\title{
Double scaling in tensor models with a quartic interaction
}

\author{
Stéphane Dartois, ${ }^{a, b}$ Razvan Gurau ${ }^{c, d}$ and Vincent Rivasseau ${ }^{b, d}$ \\ ${ }^{a}$ LIPN, Institut Galilée, CNRS UMR 7030, Université Paris 13, \\ F-93430, Villetaneuse, France \\ ${ }^{b}$ Laboratoire de Physique Théorique, Université Paris 11, \\ 91405 Orsay Cedex, France \\ ${ }^{c}$ CPHT - UMR 7644, CNRS, École Polytechnique, \\ 91128 Palaiseau Cedex, France \\ ${ }^{d}$ Perimeter Institute for Theoretical Physics, \\ 31 Caroline St. N, ON, N2L 2Y5, Waterloo, Canada \\ E-mail: stephane.dartois@ens-lyon.fr, rgurau@cpht.polytechnique.fr, \\ vincent.rivasseau@th.u-psud.fr
}

ABSTRACT: In this paper we identify and analyze in detail the subleading contributions in the $1 / N$ expansion of random tensors, in the simple case of a quartically interacting model. The leading order for this $1 / N$ expansion is made of graphs, called melons, which are dual to particular triangulations of the $D$-dimensional sphere, closely related to the "stacked" triangulations. For $D<6$ the subleading behavior is governed by a larger family of graphs, hereafter called cherry trees, which are also dual to the $D$-dimensional sphere. They can be resummed explicitly through a double scaling limit. In sharp contrast with random matrix models, this double scaling limit is stable. Apart from its unexpected upper critical dimension 6 , it displays a singularity at fixed distance from the origin and is clearly the first step in a richer set of yet to be discovered multi-scaling limits.

KeYwords: Matrix Models, 1/N Expansion, Models of Quantum Gravity

ArXiv EPRINT: 1307.5281 


\section{Contents}

1 Introduction 1

2 Matrix models 4

3 Tensor models $\quad 6$

3.1 Constructive QFT 8

4 Graph classification and the main result $\quad 14$

$\begin{array}{lll}4.1 & \text { Pruning and grafting } & 14\end{array}$

$\begin{array}{llr}4.2 & \text { Reduction } & 16\end{array}$

$\begin{array}{lll}4.3 & \text { Cherry trees } & 19\end{array}$

5 Reduced graphs amplitudes $\quad 20$

6 Proof of theorem 3 25

6.1 Bounds 25

6.2 Computation of cherry trees 26

7 The double scaling limit $\quad 26$

\section{Introduction}

Tensor models are the natural generalization of matrix models [1, 2], implementing in a consistent way statistical sums over random triangulations. The Feynman graphs of matrix models, also called ribbon graphs, are dual to maps, hence to discretizations of Riemann surfaces. Early rank- $D$ tensor models were introduced in order to generalize this remarkable property to higher dimensions $D \geq 3[3-5]$. Until recently they were difficult to handle analytically, hence were mostly studied through numerical simulations. Group field theory [6-8] is a related approach which incorporates in a common quantum field theory framework the vertex structure of early tensor models and the spin-foam amplitudes of covariant loop quantum gravity [9-11]. However early tensor models and GFTs had two major shortcomings: first they did not triangulate pseudo-manifolds with well defined $D$-homology; and even more importantly they lacked an analog of the powerful $1 / N$ expansion of matrix models [12]. This expansion structures the ordinary Feynman perturbative expansion according to the genus of the triangulated Riemann surface. It is led by the family of regular planar graphs, which can be counted exactly [13, 14]. It is this matrix $1 / N$ expansion which gave access to the single $[15,16]$ and double scaling limits $[17-19]$ of matrix models, hence to the functional integral quantization of two dimensional gravity. The perturbative expansion of string theory is also structured similarly by the genus of the string world sheet. 
The search for improved GFTs free of these two shortcomings led to the introduction of colored group field theory [20-22]. The corresponding Feynman graphs have edges of $D+1$ different colors meeting at vertices of degree $D+1$. This leads to a well-defined $D$-homology for the dual triangulations [23-25]. An associated tensorial $1 / N$ expansion was soon discovered for such colored models [26-28], providing the missing tool to structure their perturbative expansion into an interesting hierarchy. This tensorial $1 / N$ expansion is indexed by an integer called the degree. This degree is no longer a topological invariant, as was the case for matrices, but the sum over genera of jackets. Jackets are ribbon graphs embedded in the colored tensor graphs which provide $D ! / 2$ global Heegaard decompositions of the triangulation [29].

The leading order of the tensorial $1 / N$ expansion is by now well understood, and shows the same structure in any dimension $D \geq 3$. It is governed by a family of leading graphs, called melons, closely related to "stacked" triangulations of the $D$ dimensionalsphere. The corresponding single scaling critical point [30] leads to a continuous phase of branched polymers [31].

Coloring was soon recognized also as essential to formulate a general theory of unsymmetrized random tensors $[32,33]$. This theory is characterized by its invariance under the tensorial product of $D$ independent copies of the $\mathrm{U}(N)$ group. This invariance should be considered as an abstract generalization of the locality of interactions in ordinary field theory. Natural tensor interactions are polynomial invariants in the tensor coefficients, associated to bipartite colored graphs. They generalize the cyclic trace interactions of matrix models. In such so called "uncolored" models, colors reduce to a book-keeping device which tracks the position of the indices of the tensor. The tensor track program [34, 35] proposes a systematic investigation of such tensor models and of their related tensorial group field theories in order to develop the corresponding functional integral quantization of general relativity in dimension higher than two. ${ }^{1}$

Slightly breaking the $\mathrm{U}(N)$ invariance of matrix models leads to renormalizable noncommutative quantum field theories [36], such as the Grosse-Wulkenhaar (GW) model [37] whose planar sector has recently been beautifully solved [38]. The key difference between random matrix models and NCQFT's lies in the modification of the propagator. The GW propagator breaks the $\mathrm{U}(N)$ invariance of the theory in the infrared (small $N$ ) regime, but is asymptotically invariant in the ultraviolet regime (large $N$ ); hence it launches a renormalization group flow between these two regimes. Power counting in such models is entirely governed by the underlying $1 / N$ expansion (divergent graphs are the regular planar graphs), hence the corresponding matrix renormalization group can be considered the continuous version of the $1 / N$ expansion. It is remarkable that this matrix renormalization group flow is asymptotically safe [39].

In a completely analogous manner, renormalizable quantum field theories of the tensorial type have been defined through a soft breaking of the tensorial invariance of their propagator [40-42]. Renormalization is again a continuous version of the $1 / N$ expansion and the divergent graphs are the melons. The corresponding tensorial renormalization group

\footnotetext{
${ }^{1}$ Recall that the two natural parameters of tensor models, namely the coupling constant and the size $N$, can be interpreted as discrete versions of the cosmological constant and of Newton's constant.
} 
flow generically displays the fundamental physical property of asymptotic freedom [43-45]. Renormalizable GFTs which include a "Boulatov-type" propagator have also been recently defined and studied [46-48]. They incorporate as additional data discretized Lie-group valued connections over the dual triangulated pseudo-manifold, allowing to consider other metric data than the graph distance on this pseudo-manifold. Remarkably they are also asymptotically free $[49,50]$.

Other recent developments in this rapidly evolving field include extension of the $1 / N$ expansion to more general tensor models [51, 52], bounds on the number of spherical triangulations [53], investigation of the Schwinger-Dyson equations of tensor models [54-57] and of the Hopf algebraic structure of tensorial renormalization [58] and some preliminary but promising applications to statistical mechanics in presence of random geometry [59-63].

In another remarkable recent progress a rigorous non-perturbative treatment of the $1 / N$ expansion was given using an improved loop vertex expansion (LVE) [64]. ${ }^{2}$ The $\operatorname{LVE}[65,66,78]$ combines the intermediate field representation with the tree formula $[67,68]$ and replica trick of constructive field theory $[69,70]$. In contrast with ordinary perturbative expansion it is absolutely convergent, mathematically defining the correlation functions of the model as the Borel sum [71] of their perturbative series on the stable side of the coupling constant.

The LVE is in fact better adapted to the analysis of tensor models than to matrix models. Indeed it naturally allows a systematic computation of the tensorial $1 / N$ expansion. In fact it reduces rank-D tensor integration to integration over D random matrices, themselves made of replicas (one per vertex of the LVE). The leading terms, the melons, correspond to the trees of the LVE with intermediate matrix $\sigma$ fields all put to zero. The sub-leading effects in $N$ correspond to adding a finite number of loops made of Wick-contractions of these fields. $^{3}$ A mixed expansion can be written in both the coupling $\lambda$ and $N$, which allows a systematic investigation of these sub-leading effects. The situation is not identical to the random matrix case, in which all the planar terms are of the same order in $1 / \mathrm{N}$ as the LVE trees.

In this paper we use the tensorial LVE to find the equivalent of matrix double scaling for tensor models. Prior to this work, a simpler version of "matrix-like" double scaling limit for tensors was investigated in [74] and the first term beyond melons in the $1 / N$ expansion has been identified in [75]. But during the course of our investigation we had to answer new questions among which:

- How to identify the right scaling rate between $N$ and $\lambda$ and the class of graphs contributing to the double scaling? These questions are intimately intertwined.

- Is such a double expansion summable? The answer is negative in the case of matrix models but turns out to be positive for tensors in $D<6$.

\footnotetext{
${ }^{2}$ The improvement, which consists in replacing resolvents by their parametric representation, allows to factorize traces along faces of the associated graphs.

${ }^{3}$ Remark that in this representation the LVE trees form a kind of "primitive space-time" on which the intermediate matrix fields live as fluctuation fields, generating the subleading effects. This picture is quite appealing from the point of view of geometrogenesis [72, 73].
} 
Our main results are contained in Theorem 2 and 3 in section 4 , which in $D<6$ separates the next-to-leading family of cherry-trees from the rest, and in Theorem 4 in section 7 , which performs the proper double-scaling limit by summing over cherry trees. The picture emerging from our analysis is that in dimension $D<6$ the double scaling has the same susceptibility $1 / 2$ as the single scaling, hence it is possible that it leads again to a branched polymer phase. But the important points are elsewhere. First at $D=6$ or above, the double scaling incorporates larger families of graphs which may drive the system to a different phase (which remains to investigate in a future study). Second, and more important, the double scaling limit for $D<6$ seems to lead to further multiple scalings which may be probed by similar techniques. This is also left for future study, but we can speculate that the ensuing continuous phase obtained after a finite number of such steps in dimension $D=3$ or 4 is different from branched polymers.

The reader could wonder whether there is a discrepancy between our analysis and the one of [75], which finds a critical susceptibility $3 / 2$ for the next-to-leading order of the tensorial $1 / N$ expansion. In fact there is no discrepancy, since double scaling resums an infinite family of contributions, the first one of which corresponds to the one analyzed in [75]. Although the model in [75] is colored, hence not identical to ours, we have checked that the first order cherry trees of our model also give the critical susceptibility $3 / 2$ found in [75].

During preparation of this paper another parallel study lead to results similar to ours for the double scaling of colored tensor models [76]. The existence of the upper dimension 6 was found independently and on the same day by the two approaches. On one hand, the colored model treats a more general category of graphs than the uncolored quartic one, hence our analysis could be recovered as a particular case of [76]. On the other hand the methods are very different, as well as the emphasis, since the colored Bosonic model does not correspond to a stable interaction hence cannot be treated directly by the LVE. Hence the two works will be published separately.

This paper is structured as follow: in section 2 results about $1 / N$ expansion and the double scaling limit of matrix are recalled. In section 3 we introduce the basics of tensor models as well as the constructive QFT ideas that led to this work. In section 4 we introduce useful graphs definitions, manipulations and state our main results which identify the leading graphs -called cherry trees- and clarifies in which sense they dominate. Sections 5 and 6 give the proofs of our results, and the double scaling limit of the model (Theorem 4) is established in section 7.

\section{Matrix models}

We start by briefly recalling the classical results concerning the continuum limit and double scaling in matrix models.

Matrix single scaling. The non-Hermitian matrix model with a quartic interaction is the probability measure

$$
d \nu(M)=\frac{1}{Z(\lambda)}\left(\prod_{i, j=1}^{N} \frac{d M_{i j} d \bar{M}_{i j}}{2 \pi \imath}\right) e^{-N\left[\operatorname{Tr}\left(M M^{\dagger}\right)+\lambda \operatorname{Tr}\left(M M^{\dagger} M M^{\dagger}\right)\right]}
$$


where the normalization constant $Z(\lambda)$ is known as the partition function. The free energy of the model $F(\lambda)=\ln Z(\lambda)$ as well as any other correlation function (like for instance the two point function)

$$
G_{2}(\lambda)=\frac{1}{Z(\lambda)} \int\left(\prod_{i, j} N \frac{d M_{i j} d \bar{M}_{i j}}{2 \pi \imath}\right)\left(\frac{1}{N} \operatorname{Tr}\left(M M^{\dagger}\right)\right) e^{-N\left[\operatorname{Tr}\left(M M^{\dagger}\right)+\lambda \operatorname{Tr}\left(M M^{\dagger} M M^{\dagger}\right)\right]},
$$

admit an expansion in Feynman graphs. The graphs contributing to $F(\lambda)$ are ribbon graphs with quartic vertices and no external legs, while the graphs contributing to the two point function $G_{2}(\lambda)$ are ribbon graphs with quartic vertices and a marked edge. In the large $N$ limit only planar graphs survive and the planar two point function is

$$
G_{2, \text { planar }}(\lambda)=\frac{-1-36 \lambda+(1+24 \lambda)^{3 / 2}}{216 \lambda^{2}}=\sum_{n \geq 0} \frac{2}{(n+2)} \frac{3^{n}}{(n+1)}\left(\begin{array}{c}
2 n \\
n
\end{array}\right)(-2 \lambda)^{n},
$$

reproducing Tutte's counting of planar quadrangulations with a marked, oriented edge. The two point function exhibits a critical behavior at $\lambda_{c}=-\frac{1}{24}, G_{2, \text { planar }}(\lambda) \sim\left(\lambda-\lambda_{c}\right)^{1-\gamma}$, with critical exponent exponent ${ }^{4} \gamma=-\frac{1}{2}$, corresponding to pure gravity in $D=2[2]$.

Matrix double scaling. Subsequent orders in the $1 / N$ expansion of matrix models are accessed in the double scaling limit [17-19]. The $1 / N$ expansion of the two point function is indexed by the genera of the surfaces triangulated by the Feynman graphs of the model. One can thus write this expansion as:

$$
G_{2}[\lambda, N]=\sum_{h=0}^{\infty} N^{-2 h} G_{2, h}(\lambda)
$$

where $G_{2, h}(\lambda)$ is given by the sum over quadrangulations with a marked oriented edge and fixed genus $h[83]$,

$$
G_{2, h}(\lambda) \simeq \sum_{n} n^{\frac{5}{2}(h-1)}\left(\frac{\lambda}{\lambda_{c}}\right)^{n} \simeq a_{h}\left(\lambda-\lambda_{c}\right)^{\frac{3}{2}-\frac{5}{2} h} .
$$

The double scaling limit consists in taking the limit $N \rightarrow \infty$ and $\lambda \rightarrow \lambda_{c}$ in a correlated way. In fact one remarks that all $G_{2, h}$ have the same critical point $\lambda_{c}$, thus their contribution is enhanced when $\lambda \rightarrow \lambda_{c}$. Defining

$$
\kappa^{-1}=N^{5 / 4}\left(\lambda-\lambda_{c}\right)
$$

we take the limit $N \rightarrow \infty, \lambda \rightarrow \lambda_{c}$ keeping $\kappa$ fixed and we obtain the double scaling limit of the two point function

$$
G_{2}[\kappa]=\sum_{h \geq 0} \kappa^{2 h} a_{h}
$$

Unfortunately, as remarked first in [77], this expansion in $\kappa$ is not summable. First, the $a_{h}$ 's are all positive. This comes from the fact that the critical point $\lambda_{c}=-\frac{1}{24}$ is on

\footnotetext{
${ }^{4}$ This exponent is known as the string susceptibility exponent, or as the entropy exponent: it characterizes asymptotically the number of planar graphs with a fixed number of vertices.
} 
the negative real axis, hence it corresponds to an unstable potential $e^{+N\left|\lambda_{c}\right| \operatorname{Tr}\left(M M^{\dagger} M M^{\dagger}\right)}$. Second, the $a_{h}$ 's grow too fast with $h, a_{h} \simeq(2 h)$ !. This comes from the fact that in the double scaling limit one ends up summing over all the Feynman graphs at once and one encounters the usual problem that the perturbative expansion generates too many graphs.

\section{Tensor models}

The general framework of invariant tensor models is presented in detail in [32, 33, 64]. We summarize here the main points required for this paper. We deal with rank $D$ covariant tensors $\mathbb{T}_{n^{1} \ldots n^{D}}$, with $n^{1}, n^{2}, \ldots n^{D} \in\{1, \ldots N\}$ having no symmetry under permutation of their indices. The complex conjugate tensor, $\overline{\mathbb{T}}_{n^{1} \ldots n^{D}}$, is a rank $D$ contravariant tensor. They transform under the external tensor product of $D$ fundamental representations of the unitary group $\mathrm{U}(N)$

$$
\begin{aligned}
& \mathbb{T}_{a^{1} \ldots a^{D}}=\sum_{n^{1} \ldots n^{D}} U_{a^{1} n^{1}}^{(1)} \ldots U_{a^{D} n^{D}}^{(D)} \mathbb{T}_{n^{1} \ldots n^{D}} \\
& \overline{\mathbb{T}}_{\bar{a}^{1} \ldots \bar{a}^{D}}=\sum_{\bar{n}^{1} \ldots \bar{n}^{D}} \bar{U}_{\bar{a}^{1} \bar{n}^{1}}^{(1)} \ldots \bar{U}_{\bar{a}^{D} \bar{n}^{D}}^{(D)} \overline{\mathbb{T}}_{\bar{n}^{1} \ldots \bar{n}^{D}},
\end{aligned}
$$

where the indices of the complex conjugated tensor are denoted conventionally with a bar. Each unitary group acts separately on an index: the unitary operators $U^{(1)}, \ldots U^{(D)}$ are all independent (one can even consider unitary groups of different sizes $\mathrm{U}\left(N_{i}\right)$, one for each index $\left.n^{i}\right)$. We use the shorthand notation $\vec{n}$ for the $D$-tuple of integers $\left(n^{1}, \ldots n^{D}\right)$, and we consider $D \geq 3$.

Any invariant polynomial in the tensor entries can be expressed in terms of the trace invariants built by contracting in all possible ways pairs of covariant and contravariant indices in a product of tensor entries. Of particular importance in the sequel are the quartic "melonic" invariants

$$
\sum_{n \bar{n}} \mathbb{T}_{\vec{n}} \overline{\mathbb{T}}_{\vec{m}} \mathbb{T}_{\vec{m}} \overline{\mathbb{T}}_{\vec{n}} \delta_{n^{i} \bar{m}^{i}} \delta_{m^{i} \bar{n}^{i}} \prod_{j \neq i} \delta_{n^{j} \bar{n}^{j}} \delta_{m^{j} \bar{m}^{j}}
$$

They are built from four tensor entries, two $\mathbb{T}$ and two $\overline{\mathbb{T}}$, such that all indices except the one in the position $i$ are contracted between a pair $(\mathbb{T}, \overline{\mathbb{T}})$ while the indices in the position $i$ are contracted between the pairs.

The trace invariants can be represented as bipartite closed $D$-colored graphs. The graph associated to an invariant is obtained as follows:

- we represent every $\mathbb{T}_{n^{1} \ldots n^{D}}$ (respectively $\overline{\mathbb{T}}_{\bar{n}^{1} \ldots \bar{n}^{D}}$ ) by a white vertex $v$ (respectively a black vertex $\bar{v}$ )

- we represent by an edge of color $i$ the contraction of an index $n^{i}$ on $\mathbb{T}_{n^{1} \ldots n^{D}}$ with an index $\bar{n}^{i}$ of $\overline{\mathbb{T}}_{\bar{n}^{1} \ldots \bar{n}^{D}}$.

The graph of a melonic quartic invariant is represented in figure 1. Colored graphs are dual to $D$-dimensional abstract simplicial pseudo-manifolds [20, 23-25], hence the tensor models encode a theory of random triangulated higher dimensional spaces. 


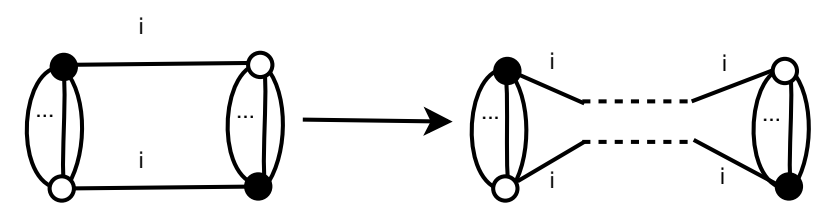

Figure 1. The Quartic Melonic Interactions.

The quartically perturbed Gaussian tensor measure with which we will deal in this paper (see [64]) is the simplest interacting tensor model, with measure

$$
\begin{gathered}
d \mu=\frac{1}{Z(\lambda, N)}\left(\prod_{\vec{n}} N^{D-1} \frac{d \mathbb{T}_{\vec{n}} d \overline{\mathbb{T}}_{\vec{n}}}{2 \pi \imath}\right) e^{-N^{D-1} S^{(4)}(\mathbb{T}, \overline{\mathbb{T}})} \\
S^{(4)}(\mathbb{T}, \overline{\mathbb{T}})=\sum_{\vec{n}} \mathbb{T}_{\vec{n}} \delta_{\vec{n} \vec{n}} \overline{\mathbb{T}}_{\vec{n}}+\lambda \sum_{i=1}^{D} \sum_{n \bar{n}} \mathbb{T}_{\vec{n}} \overline{\mathbb{T}}_{\vec{m}} \mathbb{T}_{\vec{m}} \overline{\mathbb{T}}_{\vec{n}} \delta_{n^{i} \bar{m}^{i}} \delta_{m^{i} \bar{n}^{i}} \prod_{j \neq i} \delta_{n^{j} \bar{n}^{j}} \delta_{m^{j}} \bar{m}^{j},
\end{gathered}
$$

with $Z(\lambda, N)$ some normalization constant.

Some explanations here are in order. The $D$ different quartic interactions correspond to those of figure 1, namely to the leading quartic melonic stable interactions at tensor rank (i.e. dimension) $D$. The scaling in equations (3.3) is the only scaling leading to a large $N$ limit. This is a nontrivial statement and the reader should consult [32] for its proof.

The melonic interactions turn out to be the easiest ones to decompose according to intermediate fields. Higher order stable melonic (or even submelonic) interactions can in principle be treated as well by the same method but require more intermediate fields [78]. In principle each of the $D$ interactions can have its own different coupling constant $\lambda_{i}$, but in this paper we shall treat only the symmetric case in which all $\lambda_{i}$ are equal to $\lambda$.

The two point function of the model

$$
\mathcal{G}_{2}=\frac{1}{Z(\lambda, N)} \int\left(\prod_{\vec{n}} N^{D-1} \frac{d \mathbb{T}_{\vec{n}} d \overline{\mathbb{T}}_{\vec{n}}}{2 \pi \imath}\right)\left(\frac{1}{N} \sum_{n} \mathbb{T}_{\vec{n}} \overline{\mathbb{T}}_{\vec{n}}\right) \prod_{i} \delta_{n^{i} \bar{n}^{i}} e^{-N^{D-1} S^{(4)}(\mathbb{T}, \overline{\mathbb{T}})},
$$

is well defined on the "constructive side" $\Re \lambda>0$, on which its (alternated) perturbative series is Borel summable [64]. Performing the $1 / N$ expansion of the model, either in perturbation theory or in the constructive LVE expansion $[32,64]$ one obtains that the leading order is given by melonic graphs (corresponding to the trees in the LVE expansion)

$$
\lim _{N \rightarrow \infty} \mathcal{G}_{2}=\mathcal{G}_{2, \text { melon }}=\frac{-1+\sqrt{1+8 D \lambda}}{4 D \lambda} .
$$

This corresponds to a critical constant $\lambda_{c}=-\frac{1}{8 D}$ and a susceptibility exponent $\gamma=\frac{1}{2}$. Note that, as in the matrix models case, the singularity of the leading order in the $1 / N$ expansion is on the negative real axis, corresponding to the sum with only positive signs over the class of melonic $D$ dimensional triangulations with a marked $D-1$ simplex.

The full perturbative expansion of the two point function can be reorganized as a series in $1 / N$

$$
\mathcal{G}_{2}=\mathcal{G}_{2, \text { melon }}+\sum_{h \geq 1} \frac{1}{N^{h}} \mathcal{G}_{2, h}
$$


The $1 / N$ series for tensor models is considerably more complicated than for matrix models: only some values of $h$ are allowed, the families $\mathcal{G}_{2, h}$ are complicated, and so on. The idea of the double scaling is the following. When sending $\lambda$ to $\lambda_{c}$, the melons become critical. One can then restrict to graphs $\overline{\mathcal{G}}$ with no melonic subgraphs and sum, for every such graph, the family of graphs obtained by arbitrary insertions of melons. This will lead to an expansion of the two point function

$$
\begin{aligned}
\mathcal{G}_{2} & =\mathcal{G}_{2, \text { melon }}+\sum_{\bar{G}} \frac{1}{N^{h(\bar{G})}} \frac{1}{\left(\lambda-\lambda_{c}\right)^{e(\bar{G})}} \\
& =\mathcal{G}_{2, \text { melon }}+\sum_{e \geq 1} \sum_{\bar{G}, e(\bar{G})=e}\left(\frac{1}{N^{h(\bar{G}) / e(\bar{G})}\left(\lambda-\lambda_{c}\right)}\right)^{e}
\end{aligned}
$$

where $h(\bar{G})$ is the scaling with $N$ of $\bar{G}, e(\bar{G})$ counts the number of places where we can insert melons in $\bar{G}$. We now select the family of graphs for which $h(\bar{G}) / e(\bar{G})$ is minimal. Denoting this minimal value $\alpha$, the two point function becomes

$$
\begin{aligned}
\mathcal{G}_{2}=\mathcal{G}_{2, \text { melon }}+\sum_{e \geq 1} & {\left[\sum_{\bar{G}, e(\bar{G})=e, \alpha(\bar{G})=\alpha}\left(\frac{1}{N^{\alpha}\left(\lambda-\lambda_{c}\right)}\right)^{e}\right.} \\
& \left.+\sum_{\bar{G}, e(\bar{G})=e, h(\bar{G}) / e(\bar{G})>\alpha}\left(\frac{1}{N^{h(\bar{G}) / e(\bar{G})-\alpha} N^{\alpha}\left(\lambda-\lambda_{c}\right)}\right)^{e}\right] .
\end{aligned}
$$

When tuning $\lambda$ to $\lambda_{c}$ while keeping $N^{\alpha}\left(\lambda-\lambda_{c}\right)$ fixed the last sum cancels and all the terms in the first sum admit a well defined limit. The expansion obtained in this limit is an expansion in a new double scaled parameter $x=N^{\alpha}\left(\lambda-\lambda_{c}\right)$. The rest of this paper is dedicated to establishing (3.8). As a matter of notations, in the rest of this paper we set $z=-2 \lambda$.

A very convenient way to organize the sum over graphs $\bar{G}$ is to use the constructive LVE representation of the quartic tensor model introduced in [64].

\subsection{Constructive QFT}

The main idea of constructive theory is to reorganize perturbation theory around the (classical) notion of trees, rather than around the (quantum) notion of (Feynman) graphs. Like graphs, trees have a main advantage: they show connectivity, hence allow to compute logarithms of functional integrals, the fundamental step in QFT. But since trees are much fewer than graphs, they can perform this computation in a convergent way.

Weights. The constructive reorganization of perturbation theory uses combinatoric weights defined for any pair $(G, T)$ made of a connected graph $G$ and a spanning tree $T \subset G$ [80]. The simplest definition of these weights is through Hepp sectors. A Hepp sector [81] is a total ordering of the edges of a Feynman graph. Consider a connected graph $G$ with $V$ vertices, $E$ edges and $L=E-V+1$ loop edges (independent cycles). Any spanning tree $T$ has $V$ vertices, $V-1$ edges and induces a partition of $G$ into the tree $T$ and the set $\mathcal{L}$ of the $L$ loop edges. For any Hepp sector $\sigma \in \mathfrak{S}_{E}$ of $G$ there is a single associated minimal spanning tree of $G$, denoted $T(\sigma)$, obtained by Kruskal "greedy" 
algorithm [82], that keeps recursively the edges with the smallest possible indices in $\sigma$. Defining $N(G, T)=\#\{\sigma, T(\sigma)=T\}$, the weights $w(G, T)$ are simply the percentage of sectors $\sigma$ of $G$ for which $T(\sigma)=T$ :

$$
w(G, T)=\frac{N(G, T)}{E !} .
$$

Hence these weights are rational numbers which for any graph $G$ define a probability measure on the set of its spanning trees:

$$
\sum_{T \subset G} w(G, T)=1
$$

They are symmetric in terms of relabeling of the vertices $\left\{v_{1}, \cdots v_{n}\right\}$ of the graph. A main property is their positive type integral representation

Lemma 1 ([80]).

$$
w(G, T)=\int_{0}^{1} \prod_{\ell \in T} d u_{\ell} \prod_{\ell \in G \backslash T} w_{\ell}^{T}(\{u\})
$$

where $w_{\ell}^{T}(\{u\})$ is the infimum over the $u_{\ell^{\prime}}$ parameters over the lines $\ell^{\prime}$ forming the unique path $P_{\ell}^{T}$ in $T$ joining the ends of $\ell$.

Remark that $P_{\ell}^{T}$, hence also $w_{\ell}^{T}(\{u\})$, only depends on the end vertices $i$ and $j$ of $\ell$. The representation (3.11) is called positive type because the real symmetric $V$ by $V$ matrix $W_{i, j}^{T}(u)=w_{i j}^{T}(\{u\})$ (completed by $W_{i, i}^{T}(u)=1$ on the diagonal) is of positive type for any $u_{\ell} \in[0,1]^{|T|}[67,68]$. This is what allows to bound amplitudes of the LVE representation and prove its absolute convergence [64,65]. Uniformly distributed weights $w(G, T)=1 / \chi(G)$, where $\chi(G)$ is the complexity of $G$ i.e. the number of its spanning trees, cannot be written in general as integrals such as (3.11) with positive-type matrices $w_{\ell}^{T}$, hence they do not lead to a convergent repacking of perturbation theory.

Note that each edge has an associated continuous variable: either a $u$ variable for a tree edge or a $w$ variable for a loop edge. The parameters of the tree edges in the unique path connecting the end points of a loop edge are larger than the parameter of the loop edge.

The loop vertex expansion. Reorganizing perturbation theory according to these weights allows one to decompose the Feynman amplitudes of any connected QFT quantity $S$ in terms of the spanning trees inside each Feynman graph [80]. One starts from the functional integral (in our case the tensor integral) and first performs an expansion indexed by trees

$$
S=\sum_{T} \mathcal{A}(T), \quad\left|\sum_{T} \mathcal{A}(T)\right|<\infty .
$$

This expansion is not the usual perturbative expansion and it has the main advantage that the sum over trees converges. Subsequently the contribution of each tree can be re-expanded as a sum over all graphs in which the tree is spanning

$$
\mathcal{A}(T)=\sum_{G \supset T} w(G, T) \mathcal{A}(G) \Rightarrow S=\sum_{T} \mathcal{A}(T)=\sum_{T} \sum_{G ; G \supset T} w(G, T) \mathcal{A}(G) .
$$


The subtle point is that one cannot naively exchange the sum over trees with the sum over graphs: the sum over graphs is not a summable series. However the sum over graphs is Borel summable, and the series indexed by the trees is its Borel sum. The definition of $S$ as a sum over trees (and not over graphs) is in fact its correct definition.

For Bosonic QFTs with stable interactions one must add a further twist. It turns out that the most convenient representation of $S$ as a sum over trees is obtained in terms of the intermediate field representation [65]..$^{5}$ The trees of the intermediate field representation are plane trees because the vertices of the intermediate field representation are cycles (from the point of view of the original model) made out of an arbitrary number of the ordinary propagators (hence the name "Loop Vertex Expansion"). The LVE requires decomposing the interaction into three body interactions, hence works best for quartic perturbations, but can be extended to more general stable perturbations [78].

Let $\mathcal{T}$ a plane tree with $n$ vertices labeled $1,2, \ldots n$. Consider a parameter $u_{\ell} \in[0,1]$ for each edge $\ell \in \mathcal{T}$ and a collection of $n$ replica fields $\sigma=\left\{\sigma^{1}, \cdots \sigma^{n}\right\}$, one for each vertex of the tree. Positivity of the matrix $W$ allows one to define, for every value of the $u_{\ell}$ parameters, the unique Gaussian measure $\mu^{\mathcal{T}}(u)$ over the set of random fields $\sigma$ of covariance

$$
\int d \mu^{\mathcal{T}}(u) \sigma^{i} \sigma^{j}=w_{i j}^{\mathcal{T}}(u)
$$

The LVE representation expresses $\mathcal{A}(\mathcal{T})$ as

$$
\mathcal{A}(\mathcal{T})=\int d \mu^{\mathcal{T}}(u) \quad \operatorname{Tr}\left[\prod_{q \in C(\mathcal{T})}^{\rightarrow} R\left(\sigma^{i(q)}\right)\right]
$$

where $q$ runs over the set $C(\mathcal{T})$ of the $2(n-1)$ corners of the plane tree, $\rightarrow$ means that the product is ordered along the Dyck path turning around the plane tree and $i(q)$ is the vertex reached at step $q$ in this path. The resolvent $R(\sigma)$ is defined as $R(\sigma)=[1+i H(\sigma)]^{-1}$ where $H(\sigma)$ is the Hermitian operator of the type $C^{1 / 2} \sigma C^{1 / 2}$ where $C$ is the covariance of the initial ordinary Bosonic field. Absolute convergence of the sum over trees follows from the simple fact that by Hermiticity of $H(\sigma)$ the norm of each such resolvent is bounded by $1[65,78]$.

The LVE was initially introduced to prove that the $N$-dependence of the constructive bounds for matrix QFTs reproduces the perturbative one [65]. In the matrix case however, the LVE does not by itself compute the $1 / N$ expansion. Indeed the LVE is naturally organized into trees plus loop corrections on these trees. Planar loop corrections to the LVE tree terms are not smaller than the contribution of the trees, but of the same order in $1 / N$. This is not surprising: we know that the dominant term in the matrix $1 / N$ expansion are planar graphs, which are not just simply trees. ${ }^{6}$

\footnotetext{
${ }^{5}$ For Fermionic theories the use of the intermediate field representation is not required [68], as Borel summability is not necessary as long as cutoffs are finite.

${ }^{6}$ Planar graphs are in one-to-one correspondence with well-labeled trees via the Cori-Vauquelin-Schaeffer bijection [83].
} 

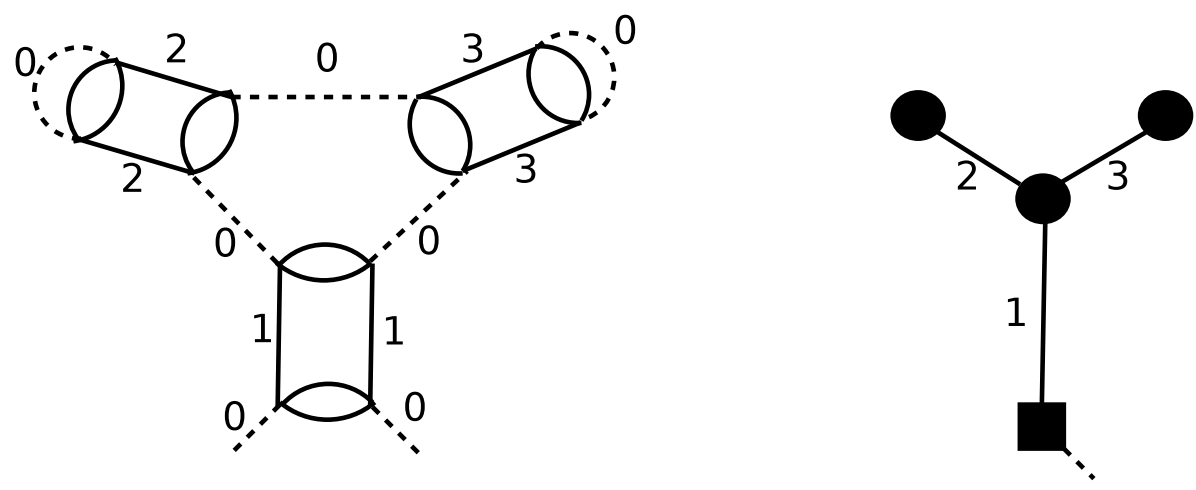

Figure 2. A LVE tree in the direct (left) and intermediate field (right) representation for a quartic interaction.

The LVE works in fact better for tensors than for matrices: its leading (tree) term directly computes the leading $1 / N$ term, at least in the case of the simple quartic model (3.3).

LVE for tensors. In the case of tensor models with quartic melonic interactions, the LVE intermediate fields are $D$ different collections of replica matrices $\sigma^{1}, \cdots \sigma^{D}$, each one acting on a particular colored strand of the ordinary tensor propagator. For tensors one needs to improve the LVE [64] by adding an additional parametric representation for the corner resolvents

$$
R=(1+i H)^{-1}=\int_{0}^{\infty} e^{-\alpha(1+i H)} d \alpha .
$$

This representation preserves the LVE convergence bounds, since $\left\|e^{-\alpha(1+i H)}\right\| \leq e^{-\alpha}$. But it has one additional remarkable property: it factorizes the action of the different colored intermediate matrix fields along well-defined colored faces, see (3.18) below. These faces are in one to one correspondence with the connected components of the tree made of edges of a single color. In particular this allows one to recover the correct scaling in $N$ of the tree amplitudes.

In order to explore subleading orders in $1 / N$ one must use the mixed representation [64]. This consists in further Taylor expanding up to fixed order $2 q$ in the coupling constant the product of resolvents. This further expansion rewrites any connected function of the theory in terms of trees decorated by up to $q$ loop edges, plus an integral remainder. ${ }^{7}$

The main advantage of the tensor loop vertex representation and of its mixed expansion as defined in [64] is that now the leading order (indexed by trees) is exactly the leading term of the $1 / N$ expansion, and the loop corrections with up to $q$ loop edges correspond to subleading terms up to order at least $N^{-q(D-2)}$ in the $1 / N$ expansion. Hence the LVE is directly suited to the study of the $1 / N$ expansion for tensors. Nevertheless not all loop corrections lead to the same combinatoric weights, or the same order in $1 / N$. They hide a

\footnotetext{
${ }^{7}$ This mixed expansion is closely related to the cleaning expansion introduced in $[84,85]$ to renormalize QFT in the LVE representation. It is not identical, as the cleaning expansion is organized along the Dyck path of the tree, whereas the mixed representation is not.
} 
much richer variety of multiple scalings and critical points than matrix models. This paper is devoted to define and analyze the first infinite class of such subleading terms.

The full formalism for computing any cumulant of $\mu$ in (3.3), i.e. all the Schwinger functions of the corresponding interacting random tensor theory has been developed in detail in [64]. It involves plane trees with ciliated vertices. The ciliated vertices correspond to external faces, and in the case of many external legs the formalism requires some heavy notations. For pedagogical reasons in this paper we shall treat only the two point function $\mathcal{G}_{2}$ of the model, which writes as a sum over plane trees $\mathcal{T}$ with a single ciliated vertex. The extension of our results to the free energy and correlation functions of arbitrary order is direct and left as an exercise.

The LVE expansion of the two point function is

$$
\mathcal{G}_{2}=\sum_{n \geq 1} \frac{(-\lambda)^{n-1}}{n !} \sum_{i_{1}, \mathcal{T}} \mathcal{A}(\mathcal{T}),
$$

where $i_{1}$ runs over the labels 1 to $n$ and $\mathcal{T}$ runs over the plane trees with $n$ vertices and oriented edges having a cilium on the vertex $i_{1}$. The contribution of a tree on $\mathcal{T}$ is

$$
\begin{aligned}
& \mathcal{A}(\mathcal{T})=\frac{1}{N^{1+n(D-1)}} \int_{0}^{1}\left(\prod_{(i, j) \in \mathcal{T}} d u_{i j}\right) \int d \mu_{w_{i j}^{\mathcal{T}}(u) 1 \otimes D}(\sigma) \\
& \int\left(\prod_{q=1}^{2 n-2+1} d \alpha_{q}\right) e^{-\sum_{q=1}^{2 n-2+1} \alpha_{q}} \prod_{f \in \mathcal{F}(\mathcal{T})} \operatorname{Tr}\left[\prod_{q \in r\left(f^{c}\right)}^{\rightarrow} e^{-\alpha_{q} \sqrt{\frac{\lambda}{N^{D-1}}}\left(\sigma^{i(q)}-\sigma^{i(q) c_{c}}\right)}\right] .
\end{aligned}
$$

In this formula the parameters $u$ are associated to the $n-1$ edges of $\mathcal{T}$. The parameters $q$ runs over the $2(n-1)+1$ corners of the tree. In the tree $\mathcal{T}$ there are $D$ single-colored sub forests $\mathcal{F}_{c}$. The sub forest $\mathcal{F}_{c}(\mathcal{T})$ is made of all edges in $\mathcal{T}$ of color $c$, and the Dyck paths $r\left(f_{c}\right)$ around each of its connected components (including the connected components made of a unique vertex) form exactly the set of faces of color $i$. The full set of faces $\mathcal{F}(\mathcal{T})$ is the union $\bigcup_{c=1}^{D} \mathcal{F}_{c}(\mathcal{T})$.

Although the intermediate matrix fields of different colors act on different strands they remain coupled in a non-trivial way since they share common $\alpha_{q}$ parameters (one per corner) and the non trivial Gaussian measure. This means that the quartic tensor model is not just a product of $D$ independent matrix models.

The $D$ collections of intermediate matrix fields $\sigma$ are distributed according to the covariances

$$
\int d \mu_{w_{i j}^{\mathcal{T}}(u) 1 \otimes D}(\sigma) \sigma_{a b}^{(k)_{c}}\left(\sigma^{(l)_{c^{\prime} \dagger}^{\dagger}}\right)_{b^{\prime} a^{\prime}}=w_{k l}^{\mathcal{T}}(u) \delta_{a a^{\prime}} \delta_{b b^{\prime}} \delta^{c c^{\prime}} .
$$

The mixed expansion is written in terms of pairs $(\mathcal{T}, \mathcal{L})$ with $\mathcal{T}$ a plane tree rooted at the cilium and $\mathcal{L}$ a set of loop edges. A rooted plane tree decorated by loop edges is a map (i.e. a graph with an ordering of the half-edges at each vertex) with colored edges, which we denote $G=\pi_{0}(\mathcal{T}, \mathcal{L})$. A map $G$ has $c$ sets of (possibly disconnected) sub maps $G_{c}$ made of the edges of color $c$ in $G$. $G_{c}$ also includes maps made of a unique vertex with no edges. We denote the set of faces of each map $G_{c}$ by $\mathcal{F}\left(G_{c}\right)$, and the set of faces of the map $G$ by $\mathcal{F}(G)=\bigcup_{c} \mathcal{F}\left(G_{c}\right) \equiv \mathcal{F}(\mathcal{T}, \mathcal{L})$. 
Theorem 1 (The Mixed Expansion, ${ }^{8}$ [64]). The contribution of a tree $\mathcal{A}(\mathcal{T})$ admits an expansion in terms of trees decorated with a finite number of loop edges $(\mathcal{T}, \mathcal{L})$ plus a Taylor remainder

$$
\begin{aligned}
\mathcal{A}(\mathcal{T}) & =\sum_{L=0}^{p-1} \mathcal{A}^{(L)}(\mathcal{T})+\mathcal{R}^{(p)}(\mathcal{T}), \\
\mathcal{A}^{(L)}(\mathcal{T}) & =\sum_{\mathcal{L},|\mathcal{L}|=L} \sum_{c_{1} \ldots c_{L}} \mathcal{A}^{(L)}(\mathcal{T}, \mathcal{L})
\end{aligned}
$$

where $\mathcal{L}$ runs over all possible ways to decorate $\mathcal{T}$ with $L$ oriented loop edges $l_{1}=\left(j_{1}, j_{1}^{\prime}\right)$, $l_{2}=\left(j_{2}, j_{2}^{\prime}\right)$ up to $l_{L}=\left(j_{L}, j_{L}^{\prime}\right)$ and $c_{1}, \ldots c_{L}$ run over the possible colorings of the loop edges. The amplitude of a tree $\mathcal{T}$ with $L$ loop edges forming the set $\mathcal{L}$ is

$$
\mathcal{A}^{(L)}(\mathcal{T}, \mathcal{L})=N^{-1-(n+L)(D-1)+|\mathcal{F}(\mathcal{T}, \mathcal{L})|} \frac{(-\lambda)^{L}}{L !} \int_{0}^{1}\left(\prod_{(i, j) \in \mathcal{T}} d u_{i j}\right) \prod_{L=1}^{s} w_{j_{L} j_{L}^{\prime}}^{\mathcal{T}}(u) .
$$

Remark that the power of $N$ exactly vanishes for melonic graphs which have $L=0$ and $|\mathcal{F}(\mathcal{T}, \emptyset)|=D+(D-1)(n-1)$. The rest term is

$$
\begin{aligned}
\mathcal{R}^{(p)}(\mathcal{T})= & \int_{0}^{1} d t(1-t)^{p-1}\left[N^{-1-(n+p)(D-1)} \frac{(-\lambda)^{p}}{(p-1) !} \sum_{\mathcal{L},|\mathcal{L}|=p} \sum_{c_{1} \ldots c_{p}} \int_{0}^{1}\left(\prod_{(i, j) \in \mathcal{T}} d u_{i j}\right)\right. \\
& \times \prod_{s=1}^{p} w_{j_{s} j_{s}^{\prime}}^{\mathcal{T}}(u) \int d \mu_{w_{i j}^{\mathcal{T}}(u) 1 \otimes D}(\sigma) \int_{0}^{\infty}\left(\prod_{q=1}^{2 n-2+2 p+1} d \alpha_{q}\right) e^{-\sum_{q=1}^{2 n-2+2 p+1} \alpha_{q}} \\
& \left.\times \prod_{f^{c} \in \mathcal{F}(\mathcal{T}, \mathcal{L})} \operatorname{Tr}\left[\prod_{q \in q\left(f^{c}\right)}^{\rightarrow} e^{\left.-\alpha_{q} \sqrt{t} \sqrt{\frac{|\lambda|}{N^{D-1}}}\left(\sigma^{i(q)}\right)_{c}-\sigma^{i(q) c_{c} \dagger}\right)}\right]\right]
\end{aligned}
$$

The term $\sum_{L=0}^{p-1} \mathcal{A}^{(L)}(\mathcal{T})$ is a polynomial hence obviously analytic in $\lambda$. Furthermore the rest term in the mixed expansion is a convergent series in $\mathcal{T}$, hence defines a function analytic in the heart-shaped domain $\lambda=e^{i \phi}|\lambda|,|\lambda| \leq$ const. $\cos ^{2} \frac{\varphi}{2}$. In this domain the following bounds hold [64]:

$$
\begin{aligned}
\left|\mathcal{A}^{(q)}(\mathcal{T})\right| & \leq K \frac{|\lambda|^{q}}{N^{q(D-2)}} \frac{(2 n+2 q-2) !}{q !(2 n-2) !} \\
\left|\mathcal{R}^{(p)}(\mathcal{T})\right| & \leq K \frac{1}{\left(\cos \frac{\varphi}{2}\right)^{2 n+2 p-1}} \frac{|\lambda|^{p}}{N^{p(D-2)}} \frac{(2 n+2 p-2) !}{(p-1) !(2 n-2) !}
\end{aligned}
$$

with $K$ some constant depending only on $D$.

The map $G=\pi_{0}(\mathcal{T}, \mathcal{L})$ has at most $D+(D-1)(n-1)+L$ faces. This explains the bounds on the remainder and ensures that all the terms up to order $N^{-p(D-2)}$ are captured

${ }^{8}$ This expansion is called the mixed expansion because it is at the same time an expansion in $\lambda$ and an expansion in $1 / N$. More precisely, being an expansion in $\frac{\lambda}{N^{D-2}}$, one can use it to establish the Borel summability of the free energy, or alternatively, one can use it to establish its $\frac{1}{N}$ expansion at all orders. 
by summing up the contributions $\mathcal{A}^{(L)}(\mathcal{T}, \mathcal{L})$ with at most $p$ loops

$$
\begin{aligned}
\mathcal{G}_{2}= & \sum_{n \geq 1} \frac{(-\lambda)^{n-1}}{n !} \sum_{i_{1}, \mathcal{T}} \sum_{L=0}^{p} \sum_{\mathcal{L},|\mathcal{L}|=L} \sum_{c_{1} \ldots c_{L}} N^{-1-(n+L)(D-1)+|\mathcal{F}(\mathcal{T}, \mathcal{L})|} \\
& \times \frac{(-\lambda)^{L}}{L !} \int_{0}^{1}\left(\prod_{(i, j) \in \mathcal{T}} d u_{i j}\right) \prod_{L=1}^{s} w_{j_{L} j_{L}^{\prime}}^{\mathcal{T}}(u)+O\left(N^{-p(D-2)}\right) .
\end{aligned}
$$

Note that a map $G=\pi_{0}(\mathcal{T}, \mathcal{L})$ might turn up to have less than $D+(D-1)(n-1)+L$ faces, in which case it contributes to an order lower than initially expected. The sum in (3.24) can be reorganized in terms of maps. Indeed, the contributions of couples $(\mathcal{T}, \mathcal{L})$ coming from the same $\mathcal{T}$ but such that the sets $\mathcal{L}$ differ just by a permutation of the edges are equal and add up to cancel the $1 / L$ ! in (3.24). Furthermore, the same map $G$ is obtained by completing any of its spanning trees by an appropriate set of loop edges (i.e. $\pi_{0}$ is onto but not one to one). However, the factors $w(G, \mathcal{T})$ for a fixed $G$ add up to 1 , hence the sum in (3.24) can be rewritten in terms of maps $G\left(i_{1}, n, L\right)$ having $n$ vertices labelled $1,2, \ldots n$, rooted at $i_{1}$ and having $n-1+L$ colored oriented edges

$$
\begin{aligned}
\mathcal{G}_{2}= & \sum_{L=0}^{p} \sum_{n \geq 1} \frac{(-\lambda)^{n+L-1}}{n !} \sum_{i_{1}, G\left(i_{1}, n, L\right)} N^{-1-(n+L)(D-1)+\left|\mathcal{F}\left(G\left(i_{1}, n, L\right)\right)\right|} \\
& +O\left(N^{-p(D-2)}\right) .
\end{aligned}
$$

Finally, grouping together the maps which differ just by a relabeling of the vertices or the orientation of the edges we get a sum over rooted maps with $n$ unlabeled vertices and $n-1+L$ colored unoriented edges,

$$
\begin{aligned}
\mathcal{G}_{2}= & \sum_{L=0}^{p} \sum_{n \geq 1}(-2 \lambda)^{n+L-1} \sum_{G \text { with } n+L-1 \text { edges and } n \text { vertices }} N^{-1-(n+L)(D-1)+F(G)} \\
& +O\left(N^{-p(D-2)}\right),
\end{aligned}
$$

where $F(G)=|\mathcal{F}(G)|$. This in particular explains why $z=-2 \lambda$ is a good variable to consider in the sequel. The - signs reflects that stability of the theory at $\lambda>0$; it also means that the singularity of $\mathcal{G}_{2}^{0}(\lambda)$ is at $\lambda=\lambda_{c}=-(8 D)^{-1}$, hence on the negative side of the real axis, which is not in the heart-shaped constructive domain of convergence. This major problem is not tackled in this paper but kept for future study.

\section{Graph classification and the main result}

\subsection{Pruning and grafting}

We can work thus either at the constructive level, which requires the LVE trees or at the perturbative with maps $G$. From now on we no longer care about the remainder term $\mathcal{R}$ : we sent $p \rightarrow \infty$ in (3.26) and ignore the fact that the perturbative sum over $L$ from 0 to $\infty$ is not summable. 


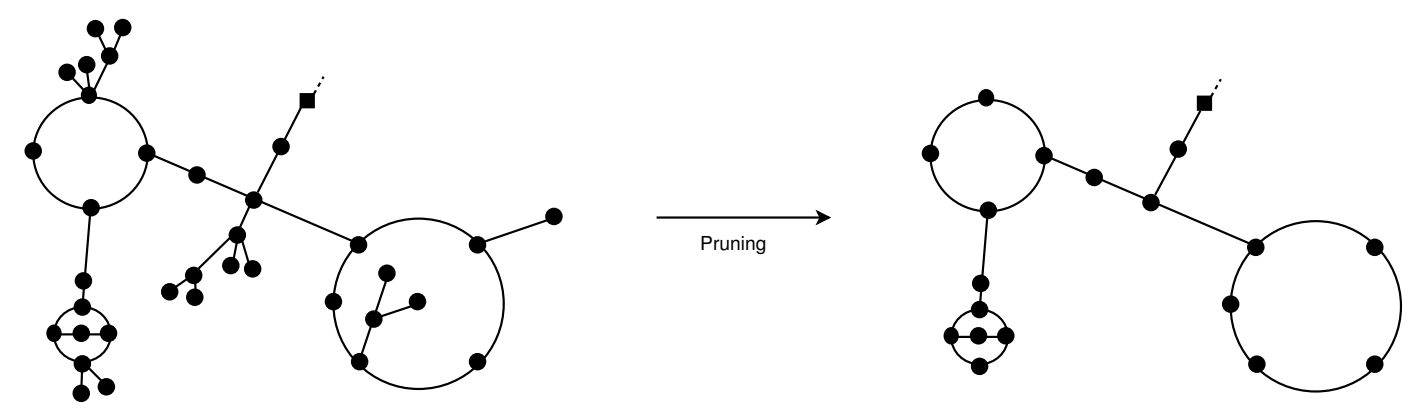

Figure 3. Pruning.

From now on we denote $V(G)=n$ the number of vertices, $E(G)=n-1+L$ the number of edges and $F(G)$ the number of faces of the map $G$. The ciliated vertex in $G$ corresponds to the external faces of the two point function $\mathcal{G}_{2}$.

To every map $G$ we will associate a map $\bar{G}$ which captures all its essential characteristics. The map $\bar{G}$ is obtained through the operations of pruning and reduction defined below. There exists an infinity of maps $G$ which correspond to the same $\bar{G}$. All such $G$ sum together and yield a contribution associated to $\bar{G}$. The perturbative series can then be re-indexed in terms of $\bar{G}$ and the double scaling limit is analyzed in terms of $\bar{G}$. Our classification is closely related to Wright's approach $[86,87]$ to the enumeration of labelled graph with a fixed number of loop edges.

We now remove iteratively all non-ciliated vertices of coordination one of $G$. This is called pruning. For simplicity, in the sequel we shall refer to maps as graphs.

Definition 1. A reducible leaf of $G$ is a vertex of $G$ of coordination 1 which is not the ciliated vertex. The pruned graph $\tilde{G}$ associated to $G$ is obtained by removing inductively all reducible leaves and their unique attaching edge.

The pruned graph is therefore obtained by removing from $G$ all rooted tree subgraphs with only reducible leaves, see figure 3 . Again the map $\pi_{1}: G \rightarrow \pi_{1}(G)=\tilde{G}$ is onto but not one to one. Remark that the pruned graph $\tilde{G}$ is never empty, as it contains at least the ciliated vertex. It also contains $L$ independent cycles, like $G$. Remark also that pruning is compatible with the coloring: the initial graph $G$ is colored, and the corresponding pruned graph $\tilde{G}$, which is a subgraph of $G$, is also colored.

The "inverse" operation of pruning is grafting: the initial graph $G$ is obtained by grafting some (possibly empty) rooted plane tree on each corner of the pruned graph $\tilde{G}$ (see figure 4). Considering all the possible graftings on $\tilde{G}$ reconstructs the entire family of graphs $G$ which reduces to $\tilde{G}$ by pruning.

Remark that the pruned graph $\tilde{G}$ has fewer vertices and fewer edges than $G$. However, at every step in the pruning process the number of edges and vertices of the graph decreases by 1 , hence $\tilde{G}$ and $G$ have the same number of cycles

$$
L(\tilde{G})=E(\tilde{G})-V(\tilde{G})+1=E(G)-V(G)+1=L(G) .
$$

Furthermore, all the graphs $G$ corresponding to the same pruned graph $\tilde{G}$ have the same scaling in $N$. Indeed, when deleting an univalent vertex (and the edge connecting it 


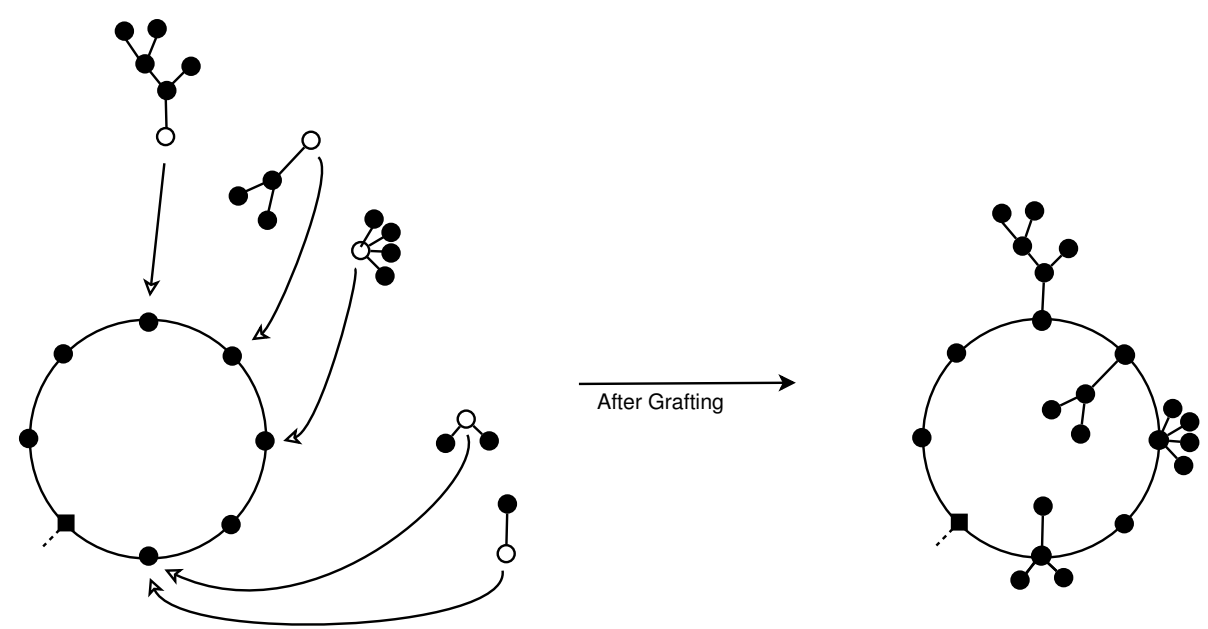

Figure 4. Grafting.

to the rest of the graph, say of color $c$ ) the number of faces of the graph decreases by $D-1$, as all the faces of color $c \neq c^{\prime}$ containing the vertex are deleted, but the face of color $c$ is not. Hence

$$
-1-(E(G)+1)(D-1)+F(G)=-1-(E(\tilde{G})+1)(D-1)+F(\tilde{G}) .
$$

Definition 2. $A$ one particle irreducible component $\tilde{G}_{k}$ (1PI) of a pruned graph $\tilde{G}$ is a maximal (non empty) connected set of edges, together with their attached vertices, which cannot be separated into two connected components by deleting one of its edges. The ciliated vertex can either be part of a one particle irreducible component, or not, in which case it is called bare. An irreducible component of a pruned graph $\tilde{G}$ is either a one particle irreducible component $\tilde{G}_{k}$, or the ciliated vertex if it is bare.

Remark that since each one particle irreducible component must include at least one loop edge (i.e. it must have at least a cycle), the number $p(\tilde{G})$ of irreducible components in a pruned graph $\tilde{G}$ with $L$ loops is at least 1 and at most $L+1$, and if $p(\tilde{G})=L+1$, the ciliated vertex must be bare.

\subsection{Reduction}

We now remove all non-ciliated vertices of coordination 2. This is called reduction.

Definition 3. A vertex of a pruned graph is called essential if it is of degree strictly greater than 2 or if it is the ciliated vertex. A bar of a pruned graph is a maximal chain of edges with internal vertices all of degree 2. The number of essential vertices and of bars of a pruned graph $\tilde{G}$ will be noted $V^{e}(\tilde{G})$ and $B(\tilde{G})$.

If we picture each bar of a pruned graph $\tilde{G}$ as a (fat) edge, we obtain a new graph $\bar{G}$ associated to $\tilde{G}$, which is made of $V(\bar{G})=V^{e}(\tilde{G})$ vertices plus $E(\bar{G})=B(\tilde{G})$ (fat) edges between them (see figure 5). It has still the same number $L(\bar{G})=L(\tilde{G})=L(G) \equiv L$ of independent cycles as $\tilde{G}$ and $G$, and every tree of $\bar{G}$ has $E(\bar{G})-L$ edges. 


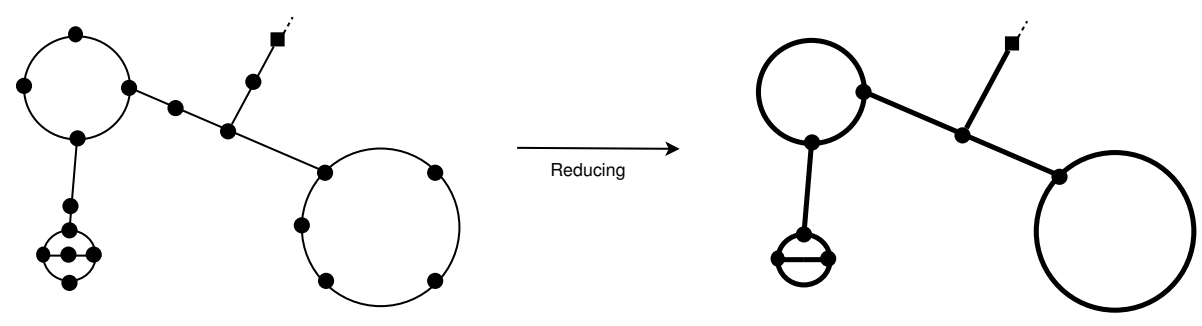

Figure 5. Reduction of a pruned graph.

Lemma 2. We have $E(\bar{G}) \leq 3 L-1$, and $V(\bar{G}) \leq 2 L$. Moreover $E(\bar{G})=3 L-1$ implies that every vertex of $\bar{G}$ is of degree 3, except the ciliated vertex $v_{c}$ which is of degree 1 .

Proofs. Let $d_{v}$ be the degree of vertex $v \in \bar{G}$. We have $d_{v} \geq 3$ for any $v$ except possibly the ciliated vertex, with degree $d_{c}$. Furthermore $V(\bar{G})-1+L=E(\bar{G})$, as $\bar{G}$ can always be decomposed into a tree of $V(\bar{G})-1$ edges plus a set of $L$ loop edges. We have

$$
2 E(\bar{G})=\sum_{v} d_{v} \geq 3(V(\bar{G})-1)+d_{c}=3(E(\bar{G})-L)+d_{c}
$$

which proves that $E(\bar{G}) \leq 3 L-d_{c} \leq 3 L-1$. Equality can happen only if $d_{v}=3$ for $v \neq v_{c}$ and $d_{c}=1$. Finally since $\bar{G}$ is connected $E(\bar{G})=V(\bar{G})-1+L$, hence $E(\bar{G}) \leq 3 L-1$ implies $V(\bar{G}) \leq 2 L$.

If a chain of edges of $\tilde{G}$ which we reduce is made of edges all with the same color $c$, we color the new fat edge in $\bar{G}$ with $c$. If on the contrary the chain of edges contained at least two edges of two different colors, we will call the fat edge multicolored, and we will associate to it an index $m$.

Definition 4. A reduced graph $\bar{G}$ is a regular graph with one ciliated vertex, such that all other vertices have coordination at least 3, plus the choice of a color $c$ or a label $m$ on any of its edges. Forgetting the labels, one gets an associated unlabeled reduced graph.

The reduction operation $\pi_{2}$ is now well-defined from the category of (colored) pruned graphs to the category of reduced graphs. The map $\pi_{2}: \tilde{G} \rightarrow \pi_{2}(\tilde{G})=\bar{G}$ is onto but not one to one. The reverse of the reduction map, called expansion, expands any edge into an arbitrarily long chain with degree 2 intermediate vertices, and sums over colorings compatible with the colors or the label $m$.

We denote $E^{m}(\bar{G})$ the number of multicolored edges of the reduced graph $\bar{G}$. We define the (possibly disconnected) graph $\bar{G}_{c}$ as the subgraph of $\bar{G}$ made of all the vertices of $\bar{G}$ and all the edges of color $c$. As $\bar{G}_{c}$ is a map, it has a certain number of faces, $F\left(\bar{G}_{c}\right)$, and a total genus $g\left(\bar{G}_{c}\right)$ (i.e. the sum of the genera of the connected components of $\bar{G}_{c}$ ). We denote the number of cycles of $\bar{G}_{c}$ by $L\left(\bar{G}_{c}\right)$.

Lemma 3. The scaling with $N$ of all the pruned graphs associated to the reduced graph $\bar{G}$ is

$$
-D L(\bar{G})+2 \sum_{c} L\left(\bar{G}_{c}\right)-2 \sum_{c} g\left(\bar{G}_{c}\right)
$$


Proofs. The scaling with $N$ of a pruned graph $\tilde{\mathcal{G}}$ is

$$
-1-(E(\tilde{G})+1)(D-1)+F(\tilde{G}) .
$$

When deleting a bivalent vertex on an unicolored edge of color $c$ the number of edges of the graph decreases by 1 and the number of faces by $D-1$ (one for each $c^{\prime} \neq c$ ). On the contrary, when deleting all the bivalent vertices on a multicolored edge with $p$ intermediate vertices, the number of edges decreases by $p$, and the number of faces by $D-2+(D-1)(p-1)$, hence the scaling with $N$ writes in terms of the data of the reduced graph as

$$
-1-(E(\bar{G})+1)(D-1)-E^{m}(\bar{G})+\sum_{c} F\left(G_{c}\right) .
$$

The graphs $G_{c}$ have $C\left(G_{c}\right)$ connected components, $V\left(G_{c}\right)$ vertices, $E\left(G_{c}\right)$ edges and total genus $g\left(G_{c}\right)$, hence the scaling with $N$ writes

$$
\begin{aligned}
& -1-(E(\bar{G})+1)(D-1)-E^{m}(\bar{G}) \\
& +\sum_{c}\left(-V\left(G_{c}\right)+E\left(G_{c}\right)+2 C\left(G_{c}\right)-2 g\left(G_{c}\right)\right) .
\end{aligned}
$$

The number of connected components of $G_{c}$ can be computed in terms of the number of cycles, $E\left(G_{c}\right)=V\left(G_{c}\right)-C\left(G_{c}\right)+L\left(G_{c}\right)$, and we get

$$
\begin{aligned}
- & -(E(\bar{G})+1)(D-1)-E^{m}(\bar{G}) \\
& +\sum_{c}\left[-V\left(G_{c}\right)+E\left(G_{c}\right)+2\left(V\left(G_{c}\right)-E\left(G_{c}\right)+L\left(G_{c}\right)\right)-2 g\left(G_{c}\right)\right] \\
= & -1-(E(\bar{G})+1)(D-1)-E^{m}(\bar{G}) \\
& +\sum_{c}\left(V\left(G_{c}\right)-E\left(G_{c}\right)+2 L\left(G_{c}\right)-2 g\left(G_{c}\right)\right) .
\end{aligned}
$$

As $V\left(G_{c}\right)=V(\bar{G})$ and $E(\bar{G})=E^{m}(\bar{G})+\sum_{c} E\left(G_{c}\right)$ we rewrite this as

$$
-1-(E(\bar{G})+1)(D-1)-E(\bar{G})+D V(\bar{G})+\sum_{c}\left(2 L\left(G_{c}\right)-2 g\left(G_{c}\right)\right),
$$

and using $E(\bar{G})=V(\bar{G})-1+L(\bar{G})$ the lemma follows.

Summing the family of graphs which, through pruning and reduction, lead to the same reduced graph $\bar{G}$ we obtain the amplitude of $\bar{G}$.

Theorem 2. The amplitude of a reduced graph $\bar{G}$ is

$$
\begin{aligned}
\mathcal{A}(\bar{G})= & N^{-D L(\bar{G})+2 \sum_{c} L\left(\bar{G}_{c}\right)-2 \sum_{c} g\left(\bar{G}_{c}\right)} T(D z)^{1+2 E(\bar{G})} \\
& \left(\frac{z}{1-z T^{2}(D z)}\right)^{\sum_{c} E\left(\bar{G}_{c}\right)}\left(\frac{D(D-1) z^{2} T^{2}(D z)}{\left[1-D z T^{2}(D z)\right]\left[1-z T^{2}(D z)\right]}\right)^{E^{m}(\bar{G})} .
\end{aligned}
$$

The proof of this theorem is presented in section 5 .

A further simplification comes from the fact that the 1PR bars of $\bar{G}$ do not in fact need any label $c$ or $m$. This comes from the fact that such graphs have the same scaling 


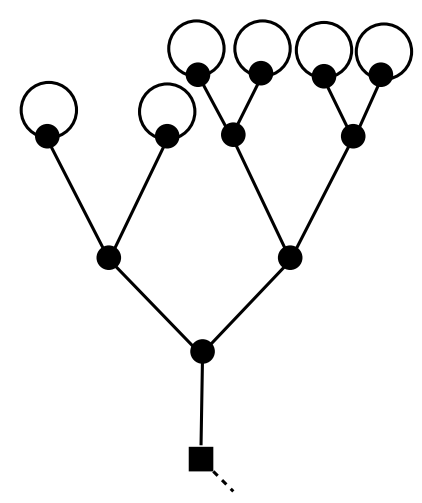

Figure 6. An example of cherry tree.

in $N$, but the graphs with colored $1 P R$ edges are suppressed in the double scaling limit: one can chose to group together or not the graphs which differ only by the label of their 1PR bars without changing the double scaling limit. Indeed, if one combines together all the reduced graphs differing only by the labels of the 1PR edges (which in this case we call free) one gets a contribution

$$
\begin{aligned}
& N^{-D L(\bar{G})+2 \sum_{c} L\left(\bar{G}_{c}\right)-2 \sum_{c} g\left(\bar{G}_{c}\right)} T(D z)^{1+2 E(\bar{G})} \\
& \left(\frac{z}{1-z T^{2}(D z)}\right)^{\sum_{c} E\left(\bar{G}_{c}\right)}\left(\frac{D(D-1) z^{2} T^{2}(D z)}{\left[1-D z T^{2}(D z)\right]\left[1-z T^{2}(D z)\right]}\right)^{E^{\mathrm{m}}(\bar{G})} \\
& \left(\frac{D z}{\left[1-D z T^{2}(D z)\right]}\right)^{E^{\mathrm{free}}(\bar{G})}
\end{aligned}
$$

having the same critical behavior as (4.10), where $E^{\text {free }}(\bar{G})$ denotes the number of free edges of $\bar{G}$ and $E^{\mathrm{m}}(\bar{G})$ the number of multicolored edges in $\bar{G}$.

\subsection{Cherry trees}

Definition 5. A reduced graph with $L(\bar{G})$ loops and one cilium is called a cherry tree (or, in short a cherry) if $E(\bar{G})=3 L(\bar{G})-1, L(\bar{G})=\sum_{c} L\left(\bar{G}_{c}\right)$, and $V\left(G_{c}\right)=C\left(G_{c}\right)$.

Remark that such a graph is a rooted binary tree made of multicolored edges, with $L(\bar{G})+1$ univalent vertices (the leaves and the root) and $L(\bar{G})-1$ trivalent vertices decorated by one self loop of color $c=1, \ldots D$ on each of its leaves. Indeed, $E(\bar{G})=3 L(\bar{G})-1$ implies that the root vertex is univalent and all other vertices are trivalent, $V\left(G_{c}\right)=C\left(G_{c}\right)$ implies that all the connected components of $G_{c}$ have exactly one vertex, $L(\bar{G})=\sum_{c} L\left(\bar{G}_{c}\right)$ and the fact that all vertices except the root are trivalent implies on one hand that $L(\bar{G})$ vertices are decorated by self loops of a fixed color, and on the other that the graph obtained by erasing these self loops has no more cycles and is connected, hence is a tree (see figure 6 and 7 ).

The mixed pruned expansion for the two-point function then splits into two parts, the sum over cherry trees and the rest, which is the sum over all other graphs:

$$
\mathcal{G}_{2}^{L}(z, N)=\mathcal{G}_{2, \text { cherry }}^{L}(z, N)+\mathcal{G}_{2, \text { rest }}^{L}(z, N) \text {. }
$$



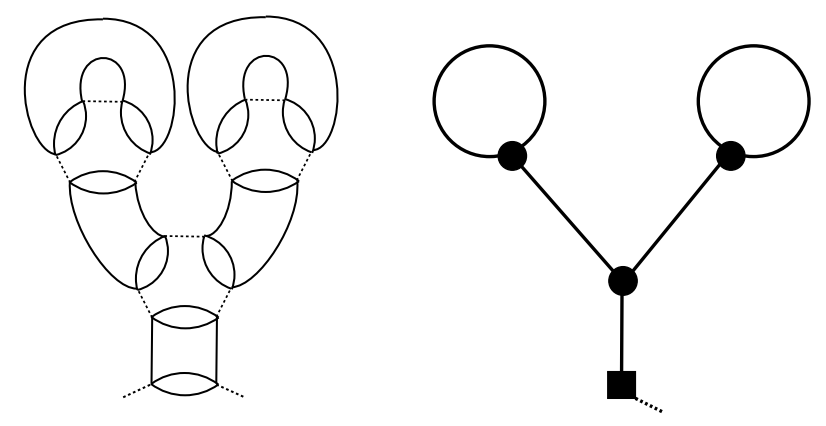

Figure 7. A cherry tree in the direct and LVE representations.

The next step is to change $z$ to the rescaled variable $x=N^{D-2}\left(z_{c}-z\right)$ with $z_{c}=(4 D)^{-1}$,

$$
\mathcal{G}_{2, \text { cherry }}^{L}(z, N)=\mathcal{G}_{2, \text { cherry }}^{L, x}(N) ; \mathcal{G}_{2, \text { rest }}^{L}(z, N)=\mathcal{G}_{2, \text { rest }}^{L, x}(N)
$$

and to consider now at fixed $L$ and $x$ the asymptotic expansion of these quantities when $N \rightarrow \infty$.

Our main result is that in this regime the cherry trees contribution $\mathcal{G}_{2, \text { cherry }}^{L, x}(N)$ has a leading term proportional to $N^{1-D / 2}$ with a coefficient which can be computed exactly, and that for $3 \leq D \leq 5$ this leading term dominates all the rest by at least one additional $N^{-1 / 2}$ factor:

Theorem 3 (Main Bound). For $3 \leq D \leq 5, L \geq 1$ fixed and for $x$ in some neighborhood of $x_{c}=\frac{1}{4(D-1)}$, we have

$$
\mathcal{G}_{2, \text { cherry }}^{L, x}(N)=N^{1-D / 2} \frac{8 \sqrt{D} C_{L-1}}{[16(D-1)]^{L}} x^{-L+1 / 2}+O\left(N^{1 / 2-D / 2}\right),
$$

where $C_{L-1}=\frac{1}{2 L-1}\left(\begin{array}{c}2 L-1 \\ L-1\end{array}\right)$ is the Catalan number of order $L-1$. Moreover there exists a constant $K_{L}$ such that

$$
\left|\mathcal{G}_{2, \text { rest }}^{L, x}(N)\right| \leq N^{1 / 2-D / 2} K_{L} x^{-\frac{3}{2} L+\frac{1}{2}} .
$$

Thus for $D<6$ the cherry trees yield the leading contribution in the double scaling limit $N \rightarrow \infty, z \rightarrow z_{c}, x=N^{D-2}\left(z_{c}-z\right)$ fixed. However remark that there is a certain degree of arbitrariness in the separation between cherry trees and the rest. The cherry trees are just the simplest convenient sub series displaying the leading double scaling behavior. The rest term is less singular, which means that adding part of it to the cherry trees would typically not change the nature of the singularity. However adding all graphs at once would certainly at some point meet the problem of divergence of perturbation theory. ${ }^{9}$

\section{$5 \quad$ Reduced graphs amplitudes}

We prove in this section Theorem 2 .

The family of pruned graphs associated to $\bar{G}$ is obtained by arbitrary insertions of bivalent vertices on any of its edges. The grafting operation adds arbitrary trees with colored edges independently on each of the corners of the pruned graphs. From (3.26) each edge of a graph has a weight $z$.

\footnotetext{
${ }^{9}$ Hence analytic continuation of the Borel sum might be the ultimate justification of (multiple) scalings.
} 
We denote $T(z)$ the generating function of plane trees with weight $z$ per edge

$$
T(z)=\sum_{n \geq 0} \frac{1}{2 n+1}\left(\begin{array}{c}
2 n+1 \\
n
\end{array}\right) z^{n}, \quad T(z)=1+z T^{2}(z), \quad T(z)=\frac{1-\sqrt{1-4 z}}{2 z} .
$$

As the edges of the LVE trees have a color $1, \ldots D$ on each edge, their generating function is $T(D z)$. We have

$$
T(D z)=1+D z T^{2}(D z) \Rightarrow 1-D z T^{2}(D z)=2-T(D z)=T(D z) \sqrt{1-4 D z} .
$$

Every corner of a pruned graphs represents a place where a tree can be inserted, hence has a weight $T(D z)$. Exactly $1+2 E(\bar{G})$ of the corners of the pruned graph $\tilde{G}$ appear in the reduced graph $\bar{G}$, hence the corners of $\bar{G}$ bring in total a factor

$$
T(D z)^{1+2 E(\bar{G})} .
$$

The weight of the edges of the reduced graph are obtained by summing over the number of intermediate bivalent vertices in the associated pruned graphs from 0 to infinity. Every intermediate vertex brings two new corners and a new edge, hence a factor $z T(D z)^{2}$. As a function of the label $c$ or $m$ of the edge in $\bar{G}$ we distinguish several cases:

- The edge in $\bar{G}$ has a color $c$. Then all the intermediate edges in $\tilde{G}$ have the same color, and we get a total weight

$$
\sum_{k=0}^{\infty} z^{k+1} T^{2 k}(D z)=\frac{z}{1-z T^{2}(D z)}
$$

- The edge in $\bar{G}$ has an index $m$. Then there must be at least two edges of different colors in $\tilde{G}$ which are replaced by the edge $m$, hence the weight is

$$
\begin{gathered}
\sum_{k=0}^{\infty} D^{k+1} z^{k+1} T^{2 k}(D z)-D \sum_{k=0}^{\infty} z^{k+1} T^{2 k}(D z) \\
=\frac{D z}{1-D z T^{2}(D z)}-\frac{D z}{1-z T^{2}(D z)} \\
=\frac{D(D-1) z^{2} T^{2}(D z)}{\left[1-D z T^{2}(D z)\right]\left[1-z T^{2}(D z)\right]}
\end{gathered}
$$

Note that if we were to consider the $1 \mathrm{PR}$ edges free, there weight becomes

$$
\frac{D z}{1-D z T^{2}(D z)}
$$

Putting together the scaling in Lemma 3 with the contributions of (5.3), (5.4) and (5.5) proves Theorem 2. We present here some low order examples of reduced graphs and their amplitudes. 


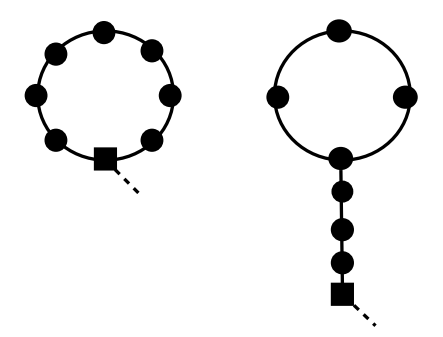

Figure 8. Two pruned graphs in the 1 loop case.

Zero loop. At the leading order in $1 / N$ only the trees with zero loops contribute. By pruning they reduce to the single ciliated vertex, which we call the graph $\bar{G}_{0} \cdot{ }^{10}$ The series of graphs $G$ corresponding to this trivial pruned graph is obtained by grafting a plane tree with colored edges on the single corner of the ciliated vertex, hence

$$
\mathcal{A}\left(\bar{G}_{0}\right)=T(D z) .
$$

reproducing the result of [64].

One loop. The first $1 / N$ corrections arise from graphs having one loop edge. There are two reduced graphs, see figure 8 showing two of the pruned graphs corresponding to each of the two reduced graphs.

Consider the leftmost graph. If the loop edge in $\bar{G}$ has a color $c$ we get an amplitude

$$
\frac{1}{N^{D-2}} T(D z)^{3} \frac{z}{1-z T^{2}(D z)},
$$

while if the loop edge is multicolored we get an amplitude

$$
\frac{1}{N^{D}} T(D z)^{3} \frac{D(D-1) z^{2} T^{2}(D z)}{\left[1-D z T^{2}(D z)\right]\left[1-z T^{2}(D z)\right]}
$$

For the graph on the right hand side the loop edge and the 1PR edge can be either unicolored or multicolored. Denoting $l$ the self loop and $t$ the vertical edge we get in each case the amplitude

$$
\begin{aligned}
& l=c ; t=c \quad \frac{1}{N^{D-2}} T(D z)^{5}\left(\frac{z}{1-z T^{2}(D z)}\right)^{2} \\
& l=c ; t=c^{\prime} \neq c \quad \frac{1}{N^{D-2}} T(D z)^{5}\left(\frac{z}{1-z T^{2}(D z)}\right)^{2} \\
& l=c ; t=m \quad \frac{1}{N^{D-2}} T(D z)^{5}\left(\frac{z}{1-z T^{2}(D z)}\right)\left(\frac{D(D-1) z^{2} T^{2}(D z)}{\left[1-D z T^{2}(D z)\right]\left[1-z T^{2}(D z)\right]}\right) \\
& l=m ; t=m \quad \frac{1}{N^{D}} T(D z)^{5}\left(\frac{D(D-1) z^{2} T^{2}(D z)}{\left[1-D z T^{2}(D z)\right]\left[1-z T^{2}(D z)\right]}\right)^{2} .
\end{aligned}
$$

\footnotetext{
${ }^{10}$ The reduction is trivial in this case as there are no two valent vertices in the pruned graph.
} 
The contribution of these graphs is obtained by summing over the choices of colors $c$ and $c^{\prime}$

$$
\begin{aligned}
& \frac{D}{N^{D-2}} T(D z)^{5}\left(\frac{z}{1-z T^{2}(D z)}\right)^{2}+\frac{D(D-1)}{N^{D-2}} T(D z)^{5}\left(\frac{z}{1-z T^{2}(D z)}\right)^{2} \\
& +\frac{D}{N^{D-2}} T(D z)^{5}\left(\frac{z}{1-z T^{2}(D z)}\right)\left(\frac{D(D-1) z^{2} T^{2}(D z)}{\left[1-D z T^{2}(D z)\right]\left[1-z T^{2}(D z)\right]}\right) \\
& +\frac{1}{N^{D}} T(D z)^{5}\left(\frac{D(D-1) z^{2} T^{2}(D z)}{\left[1-D z T^{2}(D z)\right]\left[1-z T^{2}(D z)\right]}\right)^{2} .
\end{aligned}
$$

Taking into account that

$$
1-D z T^{2}(D z)=T(D z) \sqrt{1-4 D z}, \quad T(D z) \rightarrow_{z \rightarrow(4 D)^{-1}} 2
$$

and summing up all contributions, the one loop correction to the two point function exhibits the critical behavior

$$
\mathcal{G}_{2}^{1}(z) \sim \frac{1}{N^{D-2}} \frac{D}{(D-1) \sqrt{1-4 D z}}+\frac{1}{N^{D}} \frac{1}{2(1-4 D z)} .
$$

The first term reproduces the results found in [75]: the non analytic behaviour of the first correction in $1 / N$ has a susceptibility exponent $\mathcal{G}_{2}^{1}(z) \sim\left(z_{c}-z\right)^{1-\gamma}, \gamma=+3 / 2$. This can be rewritten as

$$
\mathcal{G}_{2}^{1}(z) \sim \frac{1}{N^{\frac{D-2}{2}}}\left(\frac{\sqrt{D}}{2(D-1) \sqrt{N^{D-2}\left[(4 D)^{-1}-z\right]}}+\frac{N^{\frac{D-6}{2}}}{8 D N^{D-2}\left[(4 D)^{-1}-z\right]}\right),
$$

hence the second term is washed out for $D<6$ in the double scaling limit $N \rightarrow \infty$, $z \rightarrow(4 D)^{-1}, N^{D-2}\left[(4 D)^{-1}-z\right]$ fixed.

Two loops. There are several graphs contributing at two loops whose edges can furthermore be unicolored or multicolored. We will analyze here only some relevant examples.

Consider the reduced graph $\bar{G}_{1}$ presented in figure 9 on the left with both loops having some color $c$ and $c^{\prime}$, and the 1PR edges multicolored. It is a cherry tree. The corresponding $1 / N$ contribution is given by summing on the family of pruned graphs presented in figure 9 on the right and it is

$$
\begin{aligned}
\mathcal{A}\left(G_{1}\right) & =\frac{1}{N^{2(D-2)}} T^{11}(D z)\left(\frac{z}{\left(1-z T^{2}(D z)\right)}\right)^{2}\left(\frac{D(D-1) z^{2} T^{2}(D z)}{\left[1-D z T^{2}(D z)\right]\left[1-z T^{2}(D z)\right]}\right)^{3} \\
& =\frac{1}{N^{2(D-2)}} \frac{D^{3}(D-1)^{3} z^{8} T^{14}(D z)}{\left(1-z T^{2}(D z)\right)^{5}(1-4 D z)^{\frac{3}{2}}} \sim \frac{1}{N^{2(D-2)}(1-4 D z)^{\frac{3}{2}}}
\end{aligned}
$$

If one of the two loops is multicolored, the graph $\bar{G}_{1}^{\prime}$ has an extra $N^{-2}$ suppression in $N$ and an extra $\frac{1}{\sqrt{1-4 D z}}$ enhancement factor. Thus its amplitude is

$$
\mathcal{A}\left(\bar{G}_{1}^{\prime}\right) \sim \frac{1}{N^{2} \sqrt{1-4 D z}} \mathcal{A}\left(\bar{G}_{1}\right) \sim \frac{N^{\frac{D-6}{2}}}{\sqrt{N^{D-2}(1-4 D z)}} \mathcal{A}\left(\bar{G}_{1}\right)
$$




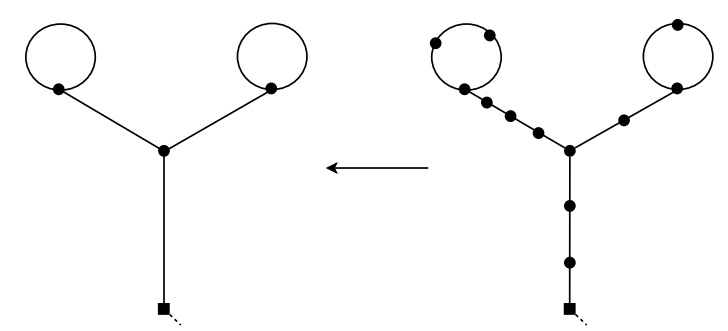

Figure 9. A pruned graph corresponding to the reduced graph $\bar{G}_{1}$.

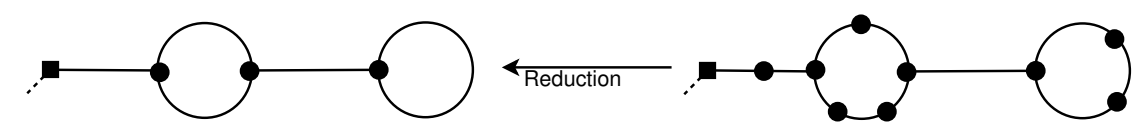

Figure 10. The reduced graph $\bar{G}_{2}$ and one pruned graph which projects onto it.

hence such reduced graphs are strictly suppressed with respect to $\bar{G}_{1}$ in the double scaling limit $N \rightarrow \infty, z \rightarrow(4 D)^{-1}, N^{D-2}\left[(4 D)^{-1}-z\right]$ fixed. Furthermore, the reduced graphs having the same structure but without the vertical $1 P R$ edge have less singular factors $\frac{1}{\sqrt{1-4 D z}}$, hence are suppressed.

A second example is the reduced graph $\bar{G}_{2}$ of figure 10. If its 1 PI bars are unicolored, its scaling with $N$ is at best $N^{-2(D-2)}$ (if the two unicolored edges in the middle have the same color $)^{11}$ the rest of its amplitude is

$$
\begin{aligned}
& T^{11}(D z)\left(\frac{z}{\left(1-z T^{2}(D z)\right)}\right)^{3}\left(\frac{D(D-1) z^{2} T^{2}(D z)}{\left[1-D z T^{2}(D z)\right]\left[1-z T^{2}(D z)\right]}\right)^{2} \\
& \quad=\frac{z^{7} D^{2}(D-1)^{2} T^{13}(D z)}{\left(1-z T^{2}(D z)\right)^{3}(1-4 D z)}
\end{aligned}
$$

and, as this graph has fewer factors $\frac{1}{\sqrt{1-4 D z}}$, it is strictly suppressed in the double scaling regime with respect to $\bar{G}_{1}$.

We now consider the case when some of the 1PI edges are multicolored. Note that if the tadpole edge is multicolored one always gets a suppressing factor $\frac{1}{N^{2} \sqrt{1-4 D z}}$ with respect to the same reduced graph where the tadpole edge is unicolored (the scaling with $N$ decreases by $N^{-2}$, while only one edge becomes critical). The most singular case is to have both 1PI edges in the middle multicolored. Such graphs scale like

$$
\frac{1}{N^{2 D-2}} \frac{1}{(1-4 D z)^{2}} \sim \frac{1}{N^{2(D-2)}(1-4 D z)^{\frac{3}{2}}} \frac{1}{N^{2} \sqrt{1-4 D z}}
$$

and again are suppressed with respect to $\bar{G}_{1}$.

Finally our last example is the graph $\bar{G}_{3}$ shown in figure 11 . As it has one fewer $1 P R$ edge than $\bar{G}_{2}$, its amplitude is less singular.

\footnotetext{
${ }^{11}$ The suppression is enhanced to $N^{-2(D-1)}$ if the two edges in the middle have different colors.
} 


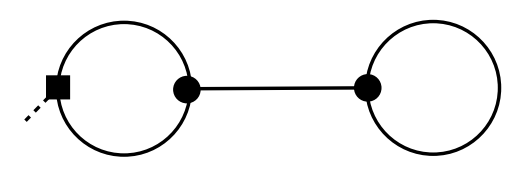

Figure 11. The reduced graph $G_{3}$.

\section{$6 \quad$ Proof of theorem 3}

\subsection{Bounds}

This section is devoted to the proof of (4.14) in Theorem 3. Recall that by Theorem 2 the amplitude of any reduced graph is

$$
\begin{aligned}
\mathcal{A}(\bar{G})= & N^{-D L(\bar{G})+2 \sum_{c} L\left(\bar{G}_{c}\right)-2 \sum_{c} g\left(\bar{G}_{c}\right)} T(D z)^{1+2 E(\bar{G})} \\
& \left(\frac{z}{1-z T^{2}(D z)}\right)^{\sum_{c} E\left(\bar{G}_{c}\right)}\left(\frac{D(D-1) z^{2} T^{2}(D z)}{\left[1-D z T^{2}(D z)\right]\left[1-z T^{2}(D z)\right]}\right)^{E^{\mathrm{m}}(\bar{G})},
\end{aligned}
$$

hence for $z$ close enough to $z_{c}$ it admits a bound

$$
|\mathcal{A}(\bar{G})| \leq K_{L} N^{-D L(\bar{G})+2 \sum_{c} L\left(\bar{G}_{c}\right)-2 \sum_{c} g\left(\bar{G}_{c}\right)}\left(z_{c}-z\right)^{-\frac{E^{\mathrm{m}}(\bar{G})}{2}} .
$$

Passing to the rescaled variable $x=N^{D-2}\left(z_{c}-z\right)$ this bound writes

$$
|\mathcal{A}(\bar{G})| \leq K_{L} N^{-D L(\bar{G})+2 \sum_{c} L\left(\bar{G}_{c}\right)-2 \sum_{c} g\left(\bar{G}_{c}\right)+\frac{D-2}{2} E^{\mathrm{m}}(\bar{G})} x^{-\frac{E^{\mathrm{m}}(\bar{G})}{2}} .
$$

Using $E(\bar{G}) \leq 3 L(\bar{G})-1$ and $E\left(\bar{G}_{c}\right)=V\left(G_{c}\right)-C\left(G_{c}\right)+L\left(G_{c}\right)$ we have

$$
E^{\mathrm{m}}(\bar{G})=E(\bar{G})-\sum_{c} E\left(\bar{G}_{c}\right) \leq 3 L(\bar{G})-1-\sum_{c} L\left(G_{c}\right)
$$

choosing $x<1$ (which includes a neighborhood of $x_{c}=\frac{1}{4(D-1)}$ ),

$$
x^{-\frac{E^{\mathrm{m}}(\bar{G})}{2}} \leq x^{-\frac{3}{2} L+\frac{1}{2}} .
$$

Furthermore, the scaling with $N$ can be bounded as

$$
\begin{aligned}
& -D L(\bar{G})+2 \sum_{c} L\left(\bar{G}_{c}\right)-2 \sum_{c} g\left(\bar{G}_{c}\right)+\frac{D-2}{2} E(\bar{G})-\frac{D-2}{2} \sum_{c} E\left(\bar{G}_{c}\right) \\
& \leq-\frac{D-2}{2}+\left(-D+\frac{D-2}{2} 3\right) L(\bar{G})+\left(2-\frac{D-2}{2}\right) \sum_{c} L\left(\bar{G}_{c}\right) \\
& =-\frac{D-2}{2}-\frac{6-D}{2} L(\bar{G})+\frac{6-D}{2} \sum_{c} L\left(\bar{G}_{c}\right),
\end{aligned}
$$

and the bound is saturated only if $E(\bar{G})=3 L(\bar{G})-1$ and $V\left(\bar{G}_{c}\right)=C\left(\bar{G}_{c}\right)$ and $g\left(G_{c}\right)=0$. If the bound is not saturated then one gets at least a suppressing factor $N^{-\frac{1}{2}}$. As $D<6$, using the fact that $\sum_{c} L\left(\bar{G}_{c}\right) \leq L(\bar{G})$, we obtain that the amplitude of any reduced graph is bounded by

$$
A(\bar{G}) \leq K_{L} N^{\frac{2-D}{2}} x^{-\frac{3}{2} L+\frac{1}{2}}
$$

and the scaling in $N$ is saturated only if $E(\bar{G})=3 L(\bar{G})-1, V\left(\bar{G}_{c}\right)=C\left(\bar{G}_{c}\right)$ and $\sum_{c} L\left(\bar{G}_{c}\right)=L(\bar{G})$, that is $\bar{G}$ is a cherry tree. For all other graphs one gets at least an extra $N^{-\frac{1}{2}}$ suppressing factor, which establishes (4.15). 


\subsection{Computation of cherry trees}

It remains to prove (4.14) by a direct computation. A full rooted binary tree is a plane tree in which every vertex has either two children or no children. The number of full binary trees with $L$ leaves is the Catalan number $C_{L-1}$. This is also the number of cherry trees with $L$ leaves since a cherry tree is just a full binary tree plus a single edge from the full binary tree to the ciliated vertex, plus addition of a tadpole with label $c$ on each leaf. Hence the map from full binary trees to cherry trees is one to one.

In the special case $L=0$, the reduced graph is just the bare ciliated vertex. It corresponds to melons, which we can consider as special degenerate cases of cherry trees. Hence

$$
\mathcal{G}_{2, \text { cherry }}^{0}=T(D z) .
$$

For $L \geq 1$ we get from Definition 5 and Theorem 2, taking into account all the possible colorings of the tadpole edges,

$$
\begin{aligned}
\mathcal{G}_{2, \text { cherry }}^{L}(z, N) & =N^{-(D-2) L} C_{L-1} D^{L} \frac{z^{5 L-2} D^{2 L-1}(D-1)^{2 L-1} T^{10 L-3}}{\left(1-z T^{2}\right)^{3 L-1}\left(1-z D T^{2}\right)^{2 L-1}} \\
& =N^{-(D-2) L} C_{L-1} \frac{z^{5 L-2} D^{3 L-1}(D-1)^{2 L-1} T^{8 L-2}}{\left(1-z T^{2}\right)^{3 L-1}(1-4 D z)^{L-\frac{1}{2}}} .
\end{aligned}
$$

In the critical regime $z \rightarrow(4 D)^{-1}$ we have $T \rightarrow 2,1-z T^{2} \rightarrow \frac{D-1}{D}, N^{D-2}(1-4 D z)=4 D x$, hence collecting all the factors we get

$$
\mathcal{G}_{2, \text { cherry }}^{L, x}(N)=N^{1-\frac{D}{2}} \frac{8 \sqrt{D} C_{L-1}}{[16(D-1)]^{L}} x^{-L+\frac{1}{2}}+O\left(N^{\frac{1}{2}-\frac{D}{2}}\right) .
$$

We recover again the susceptibility of [75] by looking at equation (6.9) at $L=1$ which give a correction in $N\left(\lambda-\lambda_{c}\right)^{-1}$ to the melonic term hence changes $\gamma_{0}$ from $1 / 2$ to $3 / 2$.

\section{The double scaling limit}

Having completed Theorem 3 we can forget, for $D<6$, all non cherry trees contributions. We can also forget the $O\left(N^{1 / 2-D / 2}\right)$ corrections in the cherry trees themselves (since they are of same order as the other discarded terms), keeping their main asymptotic behavior in (6.10) which is

$$
\overline{\mathcal{G}}_{2, \text { cherry }}^{L, x}(N)=N^{1-D / 2} \frac{8 \sqrt{D} C_{L-1}}{[16(D-1)]^{L}} x^{-L+1 / 2} .
$$

Double scaling then consists in summing these contributions over $L$ to find out the leading singularity of the sum at the critical point $x=x_{c}=\frac{1}{4(D-1)}$.

The $L=0$ contribution is the melonic contribution $T_{\text {melo }}=T(D z)$. Adding the sum over cherries gives the melonic + cherry approximation:

$$
\begin{aligned}
\overline{\mathcal{G}}_{2, \text { cherry }}^{x}(N) & =T_{\text {melo }}+8 N^{(1-D / 2)} \sqrt{D x} \sum_{L \geq 1} \frac{C_{L-1}}{[16 x(D-1)]^{L}} \\
& =T_{\text {melo }}+8 N^{(1-D / 2)} \sqrt{D x} A \sum_{L \geq 1} A^{L-1} C_{L-1},
\end{aligned}
$$


with $A=[16 x(D-1)]^{-1}$. Hence

$$
\begin{aligned}
\overline{\mathcal{G}}_{2, \text { cherry }}^{x}(N) & =T_{\text {melo }}+8 N^{(1-D / 2)} \sqrt{D x} A T(A) \\
& =T_{\text {melo }}+4 N^{(1-D / 2)} \sqrt{D x}(1-\sqrt{1-4 A}) .
\end{aligned}
$$

Since $\sqrt{1-4 A}=x^{-1 / 2} \sqrt{x-x_{c}}$

$$
\overline{\mathcal{G}}_{2, \text { cherry }}^{x}(N)=T_{\text {melo }}+4 N^{(1-D / 2)} \sqrt{D}\left(\sqrt{x}-\sqrt{x-x_{c}}\right)
$$

Substituting further $z=z_{c}-x N^{-D+2}=\frac{1}{4 D}-x N^{-D+2}$ into $T_{m e l o}$ we can reexpress

$$
\begin{aligned}
T_{\text {melo }} & =\frac{2}{1-4 D x N^{-D+2}}\left[1-2 \sqrt{D x N^{-D+2}}\right] \\
& =2-4 N^{(1-D / 2)} \sqrt{D x}+O\left(N^{2-D}\right),
\end{aligned}
$$

and we have obtained

Theorem 4. The critical point in $x$ is at $x_{c}=1 / 4(D-1)$ and the double scaling limit of the quartic tensor model two point function is

$$
\overline{\mathcal{G}}_{2, \text { cherry }}^{x}(N)=2-4 N^{(1-D / 2)} \sqrt{D\left(x-x_{c}\right)}+O\left(N^{\frac{1}{2}-\frac{D}{2}}\right) .
$$

If we rewrite everything in terms of $z$ instead of $x$, we would obtain instead

$$
\overline{\mathcal{G}}_{2, \text { cherry }}^{x}(N)=2-4 \sqrt{D\left[\left(z_{c}-z\right)-\frac{N^{2-D}}{4(D-1)}\right]}+O\left(N^{\frac{1}{2}-\frac{D}{2}}\right)
$$

where we used $x=N^{D-2}\left(z_{c}-z\right)$ and $x_{c}=1 / 4(D-1)$.

The interpretation of these formulas requires further study. Theorem 4 shows a square root singularity in $x-x_{c}$ of same critical exponent than the melonic singularity. Equation (7.10) seems to confirm that the melonic singularity has slightly moved rather than changed structure.

Triple scaling would consist in writing $\left(x-x_{c}\right) N^{\alpha}=y$ and finding a new correcting expansion in powers of $y$. It is too early to conclude on a physical interpretation, but the existence of multiple scalings suggests that in tensor models of quantum gravity space-time could emerge through a sequence of phase transitions, the first of which corresponds to a branched polymer phase.

\section{Acknowledgments}

The authors would like to thank Gilles Schaeffer for introducing them to Wright's approach to graph enumeration. In particular R.G is indebted to him for numerous discussions on the colored case of [76] which led to the clarification of many of the ideas presented in this paper.

Research at Perimeter Institute is supported by the Government of Canada through Industry Canada and by the Province of Ontario through the Ministry of Research and Innovation. 
Open Access. This article is distributed under the terms of the Creative Commons Attribution License which permits any use, distribution and reproduction in any medium, provided the original author(s) and source are credited.

\section{References}

[1] M.L. Mehta, Pure and Applied Mathematics, Volume 142: Random Matrices, Elsevier/Academic Press, (2004).

[2] P. Di Francesco, P.H. Ginsparg and J. Zinn-Justin, 2-D gravity and random matrices, Phys. Rept. 254 (1995) 1 [hep-th/9306153] [INSPIRE].

[3] J. Ambjørn, B. Durhuus and T. Jonsson, Three-dimensional simplicial quantum gravity and generalized matrix models, Mod. Phys. Lett. A 6 (1991) 1133 [INSPIRE].

[4] N. Sasakura, Tensor model for gravity and orientability of manifold, Mod. Phys. Lett. A 6 (1991) 2613 [INSPIRE].

[5] M. Gross, Tensor models and simplicial quantum gravity in $>$ 2-D, Nucl. Phys. Proc. Suppl. 25A (1992) 144 [INSPIRE].

[6] D. Boulatov, A model of three-dimensional lattice gravity, Mod. Phys. Lett. A 7 (1992) 1629 [hep-th/9202074] [INSPIRE].

[7] L. Freidel, Group field theory: an overview, Int. J. Theor. Phys. 44 (2005) 1769 [hep-th/0505016] [INSPIRE].

[8] D. Oriti, The microscopic dynamics of quantum space as a group field theory, arXiv: 1110.5606 [INSPIRE].

[9] A. Ashtekar and J. Lewandowski, Background independent quantum gravity: A Status report, Class. Quant. Grav. 21 (2004) R53 [gr-qc/0404018] [InSPIRE].

[10] C. Rovelli, Quantum Gravity, Cambridge University Press, Cambridge (2006).

[11] T. Thiemann, Modern canonical quantum General Relativity, Cambridge University Press, Cambridge (2007).

[12] G. 't Hooft, A Planar Diagram Theory for Strong Interactions, Nucl. Phys. B 72 (1974) 461 [INSPIRE].

[13] W.T. Tutte, A census of planar triangulations, Canad. J. Math. 14 (1962) 21.

[14] E. Brézin, C. Itzykson, G. Parisi and J. Zuber, Planar Diagrams, Commun. Math. Phys. 59 (1978) 35 [INSPIRE].

[15] F. David, A model of random surfaces with nontrivial critical behavior, Nucl. Phys. B 257 (1985) 543 [INSPIRE].

[16] V. Kazakov, Bilocal Regularization of Models of Random Surfaces, Phys. Lett. B 150 (1985) 282 [INSPIRE].

[17] E. Brézin and V. Kazakov, Exactly Solvable Field Theories of Closed Strings, Phys. Lett. B 236 (1990) 144 [INSPIRE].

[18] M.R. Douglas and S.H. Shenker, Strings in Less Than One-Dimension, Nucl. Phys. B 335 (1990) 635 [INSPIRE]. 
[19] D.J. Gross and A.A. Migdal, Nonperturbative Two-Dimensional Quantum Gravity, Phys. Rev. Lett. 64 (1990) 127 [INSPIRE].

[20] R. Gurau, Colored Group Field Theory, Commun. Math. Phys. 304 (2011) 69 [arXiv:0907.2582] [INSPIRE].

[21] J. Ben Geloun, J. Magnen and V. Rivasseau, Bosonic Colored Group Field Theory, Eur. Phys. J. C 70 (2010) 1119 [arXiv:0911.1719] [InSPIRE].

[22] R. Gurau and J.P. Ryan, Colored Tensor Models - a review, SIGMA 8 (2012) 020 [arXiv: 1109.4812] [INSPIRE].

[23] R. Gurau, Lost in Translation: Topological Singularities in Group Field Theory, Class. Quant. Grav. 27 (2010) 235023 [arXiv: 1006.0714] [INSPIRE].

[24] M. Pezzana, Sulla struttura topologica delle varietà compatte, Atti Sem. Mat. Fis. Univ. Modena 23 (1974) 269.

[25] M. Ferri and C. Gagliardi Crystallisation moves, Pacific J. Math. 100 (1982) 85.

[26] R. Gurau, The 1/N expansion of colored tensor models, Annales Henri Poincaré 12 (2011) 829 [arXiv: 1011.2726] [INSPIRE].

[27] R. Gurau and V. Rivasseau, The 1/N expansion of colored tensor models in arbitrary dimension, Europhys. Lett. 95 (2011) 50004 [arXiv:1101.4182] [INSPIRE].

[28] R. Gurau, The complete 1/N expansion of colored tensor models in arbitrary dimension, Annales Henri Poincaré 13 (2012) 399 [arXiv:1102.5759] [INSPIRE].

[29] J.P. Ryan, Tensor models and embedded Riemann surfaces, Phys. Rev. D 85 (2012) 024010 [arXiv:1104.5471] [INSPIRE].

[30] V. Bonzom, R. Gurau, A. Riello and V. Rivasseau, Critical behavior of colored tensor models in the large-N limit, Nucl. Phys. B 853 (2011) 174 [arXiv:1105.3122] [INSPIRE].

[31] R. Gurau and J.P. Ryan, Melons are branched polymers, arXiv:1302.4386 [INSPIRE].

[32] R. Gurau, Universality for Random Tensors, arXiv:1111.0519 [INSPIRE].

[33] V. Bonzom, R. Gurau and V. Rivasseau, Random tensor models in the large-N limit: Uncoloring the colored tensor models, Phys. Rev. D 85 (2012) 084037 [arXiv:1202.3637] [INSPIRE].

[34] V. Rivasseau, Quantum Gravity and Renormalization: The Tensor Track, AIP Conf. Proc. 1444 (2011) 18 [arXiv:1112.5104] [INSPIRE].

[35] V. Rivasseau, The Tensor Track: an Update, arXiv:1209.5284 [INSPIRE].

[36] V. Rivasseau, Non-commutative Renormalization, arXiv:0705.0705 [INSPIRE].

[37] H. Grosse and R. Wulkenhaar, Renormalization of $\phi^{4}$ theory on noncommutative $R^{4}$ in the matrix base, Commun. Math. Phys. 256 (2005) 305 [hep-th/0401128] [INSPIRE].

[38] H. Grosse and R. Wulkenhaar, Self-dual noncommutative $\phi^{4}$-theory in four dimensions is a non-perturbatively solvable and non-trivial quantum field theory, arXiv:1205.0465 [INSPIRE].

[39] M. Disertori, R. Gurau, J. Magnen and V. Rivasseau, Vanishing of $\beta$-function of Non Commutative Phi**4(4) Theory to all orders, Phys. Lett. B 649 (2007) 95 [hep-th/0612251] [INSPIRE]. 
[40] J. Ben Geloun and V. Rivasseau, A Renormalizable 4-Dimensional Tensor Field Theory, Commun. Math. Phys. 318 (2013) 69 [arXiv:1111.4997] [InSPIRE].

[41] J. Ben Geloun and E.R. Livine, Some classes of renormalizable tensor models, J. Math. Phys. 54 (2013) 082303 [arXiv:1207.0416] [INSPIRE].

[42] J. Ben Geloun, Renormalizable Models in Rank $d \geq 2$ Tensorial Group Field Theory, arXiv:1306.1201 [INSPIRE].

[43] J. Ben Geloun, Two and four-loop $\beta$-functions of rank 4 renormalizable tensor field theories, Class. Quant. Grav. 29 (2012) 235011 [arXiv:1205.5513] [INSPIRE].

[44] J. Ben Geloun and D.O. Samary, 3D Tensor Field Theory: Renormalization and One-loop $\beta$-functions, Annales Henri Poincaré 14 (2013) 1599 [arXiv: 1201.0176] [INSPIRE].

[45] J. Ben Geloun, Asymptotic Freedom of Rank 4 Tensor Group Field Theory, arXiv: 1210.5490 [INSPIRE].

[46] S. Carrozza, D. Oriti and V. Rivasseau, Renormalization of Tensorial Group Field Theories: Abelian U(1) Models in Four Dimensions, arXiv:1207.6734 [INSPIRE].

[47] D.O. Samary and F. Vignes-Tourneret, Just Renormalizable TGFT's on U(1) ${ }^{d}$ with Gauge Invariance, arXiv:1211.2618 [INSPIRE].

[48] S. Carrozza, D. Oriti and V. Rivasseau, Renormalization of an SU(2) Tensorial Group Field Theory in Three Dimensions, arXiv:1303.6772 [INSPIRE].

[49] D.O. Samary, $\beta$-functions of $\mathrm{U}(1)^{d}$ gauge invariant just renormalizable tensor models, arXiv:1303.7256 [INSPIRE].

[50] S. Carrozza, in preparation.

[51] V. Bonzom, New 1/N expansions in random tensor models, JHEP 06 (2013) 062 [arXiv: 1211.1657] [INSPIRE].

[52] S. Dartois, V. Rivasseau and A. Tanasa, The $1 / N$ expansion of multi-orientable random tensor models, arXiv:1301.1535 [INSPIRE].

[53] V. Rivasseau, Spheres are rare, arXiv:1303.7371 [INSPIRE].

[54] R. Gurau, A generalization of the Virasoro algebra to arbitrary dimensions, Nucl. Phys. B 852 (2011) 592 [arXiv:1105.6072] [INSPIRE].

[55] R. Gurau, The Schwinger Dyson equations and the algebra of constraints of random tensor models at all orders, Nucl. Phys. B 865 (2012) 133 [arXiv: 1203.4965] [InSPIRE].

[56] T. Krajewski, Schwinger-Dyson Equations in Group Field Theories of Quantum Gravity, arXiv:1211.1244 [INSPIRE].

[57] V. Bonzom, Revisiting random tensor models at large- $N$ via the Schwinger-Dyson equations, JHEP 03 (2013) 160 [arXiv: 1208.6216] [INSPIRE].

[58] M. Raasakka and A. Tanasa, Combinatorial Hopf algebra for the Ben Geloun-Rivasseau tensor field theory, arXiv:1306.1022 [INSPIRE].

[59] V. Bonzom, R. Gurau and V. Rivasseau, The Ising Model on Random Lattices in Arbitrary Dimensions, Phys. Lett. B 711 (2012) 88 [arXiv:1108.6269] [INSPIRE].

[60] D. Benedetti and R. Gurau, Phase Transition in Dually Weighted Colored Tensor Models, Nucl. Phys. B 855 (2012) 420 [arXiv:1108.5389] [INSPIRE]. 
[61] V. Bonzom, R. Gurau and M. Smerlak, Universality in p-spin glasses with correlated disorder, Journal of Statistical Mechanics: Theory and Experiment 2 (2013) 0 [arXiv:1206.5539].

[62] V. Bonzom, Multicritical tensor models and hard dimers on spherical random lattices, Phys. Lett. A 377 (2013) 501 [arXiv: 1201.1931] [INSPIRE].

[63] V. Bonzom and H. Erbin, Coupling of hard dimers to dynamical lattices via random tensors, J. Stat. Mech. 1209 (2012) P09009 [arXiv:1204.3798] [INSPIRE].

[64] R. Gurau, The 1/N Expansion of Tensor Models Beyond Perturbation Theory, arXiv: 1304.2666 [INSPIRE].

[65] V. Rivasseau, Constructive Matrix Theory, JHEP 09 (2007) 008 [arXiv:0706.1224] [INSPIRE].

[66] J. Magnen and V. Rivasseau, Constructive $\phi^{4}$ field theory without tears, Annales Henri Poincaré 9 (2008) 403 [arXiv: 0706.2457] [InSPIRE].

[67] D. Brydges and T. Kennedy, Mayer expansions and the Hamilton-Jacobi equation, J. Stat. Phys. 48 (1987) 19.

[68] A. Abdesselam and V. Rivasseau, Trees, forests and jungles: a botanical garden for cluster expansions, in Lecture Notes in Physics. Volume 446: Constructive Physics, V. Rivasseau ed., Springer Verlag, (1995) [hep-th/9409094] [INSPIRE].

[69] J. Glimm and A. Jaffe, Quantum physics. A functional integral point of view, second edition, Springer, (1987).

[70] V. Rivasseau, From perturbative to constructive renormalization, Princeton series in physics, Princeton University Press, Princeton U.S.A. (1991).

[71] A. Sokal, An improvement of Watson's theorem on Borel summability, J. Math. Phys. 21 (1980) 261 [INSPIRE].

[72] T. Konopka, F. Markopoulou and L. Smolin, Quantum Graphity, hep-th/0611197 [INSPIRE].

[73] D. Oriti, Disappearance and emergence of space and time in quantum gravity, arXiv:1302.2849 [INSPIRE].

[74] R. Gurau, The Double Scaling Limit in Arbitrary Dimensions: A Toy Model, Phys. Rev. D 84 (2011) 124051 [arXiv:1110.2460] [INSPIRE].

[75] W. Kaminski, D. Oriti and J.P. Ryan, Towards a double-scaling limit for tensor models: probing sub-dominant orders, arXiv:1304.6934 [INSPIRE].

[76] R. Gurau and G. Schaeffer, to appear.

[77] F. David, Phases of the large- $N$ matrix model and nonperturbative effects in $2-D$ gravity, Nucl. Phys. B 348 (1991) 507 [INSPIRE].

[78] V. Rivasseau and Z. Wang, Loop Vertex Expansion for Phi**2K Theory in Zero Dimension, J. Math. Phys. 51 (2010) 092304 [arXiv: 1003.1037] [INSPIRE].

[79] J. Magnen, K. Noui, V. Rivasseau and M. Smerlak, Scaling behaviour of three-dimensional group field theory, Class. Quant. Grav. 26 (2009) 185012 [arXiv:0906.5477] [INSPIRE].

[80] V. Rivasseau and Z. Wang, How to Resum Feynman Graphs, arXiv:1304.5913 [INSPIRE].

[81] K. Hepp, Théorie de la renormalisation, Springer Verlag, Berlin (1969). 
[82] J.B. Kruskal, On the Shortest Spanning Subtree of a Graph and the Traveling Salesman Problem, Proc. Amer. Math. Soc. 7 (1956) 48.

[83] G. Schaeffer, Conjugaison d'arbres et cartes combinatoires aléatoires, Université Bordeaux I, 1998.

[84] V. Rivasseau and Z. Wang, Constructive Renormalization for $\Phi_{2}^{4}$ Theory with Loop Vertex Expansion, J. Math. Phys. 53 (2012) 042302 [arXiv:1104.3443] [INSPIRE].

[85] Z. Wang, Construction of 2-dimensional Grosse-Wulkenhaar Model, PoS(CORFU2011) 057 [arXiv: 1104.3750] [INSPIRE].

[86] E. Wright, The number of connected sparsely edge graphs, J. Graph. Theor. 1 (1977) 317.

[87] E. Wright, The number of connected sparsely edged graphs. II. Smooth graphs and blocks, J. Graph. Theor. 2 (1978) 299. 